\title{
High-Energy Passive Mode-Locking of Fiber Lasers
}

\author{
Edwin Ding, ${ }^{1}$ William H. Renninger, ${ }^{2}$ Frank W. Wise, ${ }^{2}$ Philippe Grelu, ${ }^{3}$ \\ Eli Shlizerman, ${ }^{4}$ and J. Nathan Kutz ${ }^{4}$ \\ ${ }^{1}$ Department of Mathematics and Physics, Azusa Pacific University, P.O. Box 7000, Azusa, CA 91702-7000, USA \\ ${ }^{2}$ Department of Applied Physics, Cornell University, Ithaca, NY 14853, USA \\ ${ }^{3}$ Laboratoire Interdisciplinaire Carnot de Bourgogne, UMR 5209 CNRS, Université de Bourgogne, 21000 Dijon, France \\ ${ }^{4}$ Department of Applied Mathematics, University of Washington, Box 352420, Seattle, WA 98195-2420, USA
}

Correspondence should be addressed to Edwin Ding, eding@apu.edu

Received 1 October 2011; Accepted 18 January 2012

Academic Editor: Francesco Prudenzano

Copyright (๑) 2012 Edwin Ding et al. This is an open access article distributed under the Creative Commons Attribution License, which permits unrestricted use, distribution, and reproduction in any medium, provided the original work is properly cited.

\begin{abstract}
Mode-locking refers to the generation of ultrashort optical pulses in laser systems. A comprehensive study of achieving high-energy pulses in a ring cavity fiber laser that is passively mode-locked by a series of waveplates and a polarizer is presented in this paper. Specifically, it is shown that the multipulsing instability can be circumvented in favor of bifurcating to higher-energy single pulses by appropriately adjusting the group velocity dispersion in the fiber and the waveplate/polarizer settings in the saturable absorber. The findings may be used as practical guidelines for designing high-power lasers since the theoretical model relates directly to the experimental settings.
\end{abstract}

\section{Introduction}

The invention of the laser by Maiman in 1960 is a historical landmark of advanced scientific innovation. Lasers serve as lightwave sources for pulsed electromagnetic energy and have a large variety of applications, ranging from small-scale problems such as ocular surgeries and biological imaging to large-scale problems such as optical communication systems and nuclear fusion. In the context of telecommunications and broadband sources, the laser is required to robustly produce optical pulses on the scale of picoseconds or even femtoseconds. The generation of such short pulses is often referred to as mode-locking. Depending on the design of the laser cavity, mode-locking can be classified as either active or passive. The active mode-locking mechanism uses an external signal to induce a modulation on the propagating electromagnetic field inside the laser cavity, whereas the passive mode-locking mechanism relies on placing some discrete element(s) into the laser cavity which causes self-amplitude modulation of the field. This paper discusses some of the latest theoretical developments in high-power passive modelocked lasers.

A common feature to all mode-locked lasers is the intensity discrimination that is achieved by the mode-locking mechanism [1-3]. Such intensity discrimination, also known as saturable absorption, is the underlying mechanism responsible for passive mode-locking [4]. Figure 1 shows a typical passively mode-locked fiber laser that utilizes a ring configuration $[1,5,6]$. The laser cavity consists a piece of singlemode fiber (SMF), an output coupler that extracts the signal out of the cavity after each round trip, and a bandwidth-limited energy pump that compensates the energy lost during propagation. The saturable absorber considered here consists of a linear polarizer and a series of waveplates. When combined with the laser cavity polarization rotation, bandwidthlimited saturating gain, chromatic dispersion, and self-phase modulation, a uniform train of stable mode-locked pulses may be formed from white noise after a certain number of round trips. Saturable absorption can also be achieved by a variety of other physical mechanisms including nonlinear polarization rotation [7-10], nonlinear interferometry [11$14]$, graphene-based lattices $[15,16]$, and nonlinear modecoupling [17-20], but the primary focus in this paper is the saturable absorption achieved by the polarizer and waveplates.

Since its first proposed use in the early 90s, the fiber ring cavity laser mode-locked by a passive polarizer and a series of 


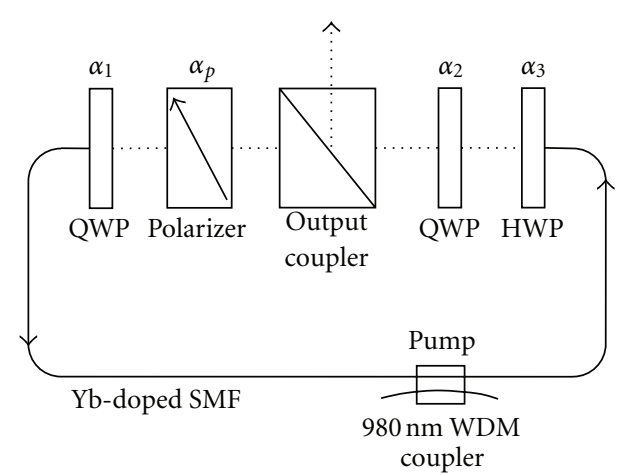

FIGURE 1: Experimental configuration of a ring laser cavity that includes quarter-waveplates (QWP), passive polarizer, half-waveplate (HWP), ytterbium-doped amplification, and output coupler. The $\mathrm{Yb}$-doped section of the fiber is fused with standard single-mode fiber and treated in a distributed fashion. The angles $\alpha_{1}, \alpha_{2}, \alpha_{3}$, and $\alpha_{p}$ of the discrete mode-locking elements can all be measured with reasonable accuracy. The dotted lines denote free space (from [6]).

quarter- and half-waveplates (see Figure 1) has become one of the most reliable and compact sources for robust ultrashort optical pulses [1, 3, 7-9]. Such fiber lasers offer major practical advantages over solid-state configurations. For example, they are relatively cheap and compact and do not require careful alignment of the optical cavity since the light is contained in a waveguide. The possibility of using fibers in short-pulse laser devices has motivated research for nearly two decades. However, limitations in the energy output have limited their impact in comparison to their solid-state counterparts. Indeed, fiber lasers have lagged well behind solidstate lasers in the key performance parameters-pulse energy and duration. Recently, new insights into pulse-propagation physics [21-23] have provided glimpses of order-ofmagnitude increases in the pulse energy and peak power in femtosecond fiber lasers. However, the design and optimization of high-performance fiber devices is impeded by the so-called multipulsing instability (MPI), which ultimately imposes a fundamental limitation on a single mode-locked pulse energy $[5,24]$.

To compete directly with solid-state technologies, it is critical to understand the limiting effects of the MPI. A number of theoretical and computational models have been introduced over the past two decades in an attempt to quantify the mode-locking dynamics in a laser cavity with a passive polarizer. The master mode-locking equation first proposed by Haus [1] is the most well-known and recognized model to date. This model, later augmented by a quintic nonlinearity to account for the experimentally observed robust nature of the mode-locked pulses $[3,6,25]$, is also known as the cubicquintic Ginzburg-Landau equation (CQGLE). In this model, the discrete laser dynamics are averaged out and replaced by a truncated Taylor expansion addressing the Kerr nonlinearity and saturable absorption. Although the CQGLE gives good qualitative descriptions of the averaged modelocking dynamics that are consistent with experiments $[5,6]$ and allows for extensive theoretical and numerical analysis [26-28], it is shown that the model fails to capture the poten- tial high-energy pulses that are practically important to the community [29].

This paper gives an overview of the theoretical models that are used to describe the pulse propagation in the laser cavity $[1,3]$. The primary focus is to exploit the system parameters with the aim of circumventing the MPI in favor of bifurcating to high-energy single-pulse solutions of the mode-locking models. The analysis and numerical simulations presented here provide mathematical insights into the underlying mode-locking dynamics and, more importantly, guidelines for experimentalists to optimize the performance in the mode-locked laser.

\section{Governing Equations}

As with all other electromagnetic phenomena, the propagation of the electric field in the laser cavity shown in Figure 1 is governed by Maxwell's equations. A multiscale asymptotic expansion of the solution to Maxwell's equation can be performed to separate the slowly varying envelope from the transverse dependence of the electric field [30,31]. To describe the propagation of the electric field envelope, the dominant physical effects must be accounted for. These include chromatic dispersion, fiber birefringence, Kerr nonlinearity, cavity attenuation, bandwidth-limited saturable gain, and the discrete effects of the waveplates and polarizer. Under certain choice of the angles $\alpha_{1}, \alpha_{2}, \alpha_{3}$, and $\alpha_{p}$, the waveplates and the polarizer provide an effective intensity discrimination (saturable absorption) to shape the electric field circulating in the cavity $[6,25]$.

2.1. Full Governing Equations. The full governing equations for modeling the pulse evolution in the laser shown in Figure 1 can be divided into two parts: (i) the intra-cavity dynamics induced by the interactions of chromatic dispersion, Kerr nonlinearity gain saturation, and so forth and (ii) the discrete application of the waveplates and polarizer after each cavity round trip. It has been shown that the intracavity evolution is described by a pair of dimensionless coupled nonlinear Schrödinger equations (CNLS) [30-33]:

$$
\begin{aligned}
& i \frac{\partial u}{\partial z}+\frac{D}{2} \frac{\partial^{2} u}{\partial t^{2}}-K u+\left(|u|^{2}+A|v|^{2}\right) u+B v^{2} u^{*}=i R_{u} \\
& i \frac{\partial v}{\partial z}+\frac{D}{2} \frac{\partial^{2} v}{\partial t^{2}}+K v+\left(A|u|^{2}+|v|^{2}\right) v+B u^{2} v^{*}=i R_{v} .
\end{aligned}
$$

In the above system, $u(z, t)$ and $v(z, t)$ represent the two orthogonally polarized electric field envelopes in an optical fiber with birefringence $K$ and are usually referred to as the fast and slow components of the electric field, respectively. The $z$ coordinate denotes the propagating distance normalized by the length of the cavity, and $t$ denotes the retarded time normalized by the full width at half-maximum of the pulse. $D$ is the averaged group velocity dispersion of the cavity and is positive for anomalous dispersion and negative for normal dispersion. The material properties of the optical fiber determine the values of the nonlinear coupling parameters $A$ 
(cross-phase modulations) and $B$ (four-wave mixing). These parameters satisfy $A+B=1$ by axisymmetry and, for the silica fiber considered, take on the specific values $A=2 / 3$ and $B=1 / 3[32,33]$. The dissipative terms $R_{u}$ and $R_{v}$, accounting for the saturable, bandwidth-limited gain (from the $\mathrm{Yb}$ doped amplification) and attenuation, take the form

$$
R_{\sigma}=G(z)\left(1+\tau \partial_{t}^{2}\right) \sigma-\Gamma \sigma,
$$

with

$$
G(z)=\frac{2 g_{0}}{1+\left(1 / e_{0}\right) \int_{-\infty}^{\infty}\left(|u|^{2}+|v|^{2}\right) d t} .
$$

Here, $g_{0}$ and $e_{0}$ are the nondimensional pumping strength and the saturating energy of the gain medium, respectively. The parameter $\tau$ characterizes the bandwidth of the pump, and $\Gamma$ measures the distributed losses caused by the output coupling and the fiber attenuation.

The discrete effect of the waveplates and passive polarizer after each cavity round trip can be modeled by the corresponding Jones matrices $[5,6]$. The standard Jones matrices for the quarter-waveplate, half-waveplate, and polarizer are given, respectively, by

$$
\begin{aligned}
& W_{\lambda / 4}=\left(\begin{array}{cc}
e^{-i \pi / 4} & 0 \\
0 & e^{i \pi / 4}
\end{array}\right), \\
& W_{\lambda / 2}=\left(\begin{array}{cc}
-i & 0 \\
0 & i
\end{array}\right), \quad W_{p}=\left(\begin{array}{ll}
1 & 0 \\
0 & 0
\end{array}\right) .
\end{aligned}
$$

These matrices are valid only when the principal axes of the devices are aligned with the fast field of the cavity. For arbitrary orientation $\alpha_{j}(j=1,2,3, p)$ shown in Figure 1, the matrices are modified according to

$$
J_{j}=R\left(\alpha_{j}\right) W R\left(-\alpha_{j}\right),
$$

where $W$ is the Jones matrix of the device given in (4) and $R$ is the rotation matrix

$$
R\left(\alpha_{j}\right)=\left(\begin{array}{cc}
\cos \alpha_{j} & -\sin \alpha_{j} \\
\sin \alpha_{j} & \cos \alpha_{j}
\end{array}\right) .
$$

The field $(u, v)^{+}$immediately after any mode-locking element $J_{j}$ can be related back to the field $(u, v)^{-}$immediately before the element by

$$
\left(\begin{array}{l}
u \\
v
\end{array}\right)^{+}=J_{j}\left(\begin{array}{l}
u \\
v
\end{array}\right)^{-} .
$$

To help make clear the modeling of the laser cavity dynamics subject to (1)-(6), consider a single round trip passage through the cavity. The propagation of the field starts immediately after the polarizer with orientation $\alpha_{p}$ for which the pulse is linearly polarized. The quarter-waveplate (with angle $\alpha_{1}$ ) to the left of the polarizer converts the polarization state from linear to elliptical, thus creating a polarization ellipse. The two polarization components $u$ and $v$ then propagate through the fiber as governed by (1). At the end of the fiber, the half-waveplate (with angle $\alpha_{3}$ ) further rotates the polarization ellipse through a certain angle. The quarterwaveplate (with angle $\alpha_{2}$ ) converts the polarization state from elliptical back to linear, and the polarizer finally aligns the field with its own principal axis.

The CNLS (1) together with Jones matrices (5) gives a full description of pulse propagation in the laser system. We first apply a fast Fourier transform to convert the temporal domain of the CNLS to the spectral domain. An adaptive step-size fourth-order Runge-Kutta algorithm is then used to propagate the initial data (in the spectral domain) over one cavity round trip. The Jones matrices of the waveplates and polarizer are then applied to the end data sequentially, and the entire procedure is repeated. The discrete application of Jones matrices after each cavity round trip acts like a filter that can be tuned to control the mode-locking behavior. Depending on their orientations, the waveplates and the polarizer can either destabilize the field propagating in the cavity or provide an effective intensity discriminating mechanism to lock it into a robust pulse. Figure 2 shows how stable, self-starting mode-locking can be achieved in the laser cavity shown in Figure 1. At a pumping strength $g_{0}=1$ (Figures 2(a) and 2(b)), the initial white noise is mode-locked into a stable pulse after about two hundred cavity round trips. When the pumping strength is increased to $g_{0}=2.7$ (Figures 2(c) and 2(d)), stable single-pulse mode-locking cannot be achieved. Specifically, the increased pumping strength broadens the frequency spectrum of the pulse, which eventually exceeds the bandwidth of the pump. In this case the initial white noise quickly evolves into the next energetically favorable state of two identical pulses. The bifurcation of a single pulse into multiple pulses (multipulsing instability (MPI)) is the main focus of this paper.

2.2. The Master Mode-Locking Equation. Although it is not difficult to simulate the full governing system (1)-(5) [21, 23, $26,34-38]$, extracting analytic results remains a mathematical challenge due to the discrete nature of the implementation of the waveplates and polarizer. This has led to the consideration of averaged evolution models that distribute all the lumped effects over the entire cavity while retaining the key mode-locking dynamics $[39,40]$. The master modelocking equation proposed by Haus [1, 7-9] was the first theoretical model used to describe the mode-locking dynamics in the ring cavity laser shown in Figure 1. This model is essentially the complex Ginzburg-Landau equation (CGLE) where a cubic nonlinearity is used to describe the action of the saturable absorber. A quintic term is usually added to the master mode-locking equation to account for the robustness of the pulses observed in experiments, and the resulting equation, generally known as the cubic-quintic GinzburgLandau equation (CQGLE), takes the form

$$
\begin{aligned}
i \psi_{z} & +\frac{D}{2} \psi_{t t}+\gamma|\psi|^{2} \psi+\nu|\psi|^{4} \psi \\
& =i g(z)\left(1+\tau \partial_{t}^{2}\right) \psi-i \delta \psi+i \beta|\psi|^{2} \psi+i \mu|\psi|^{4} \psi .
\end{aligned}
$$




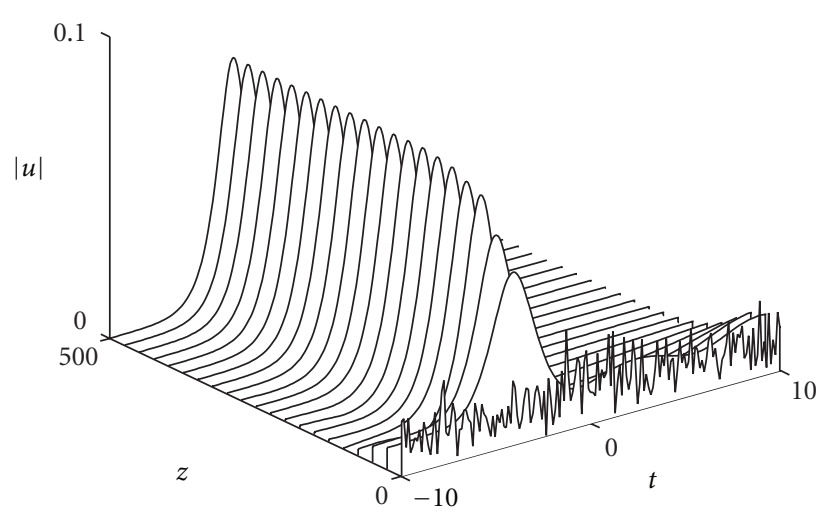

(a)

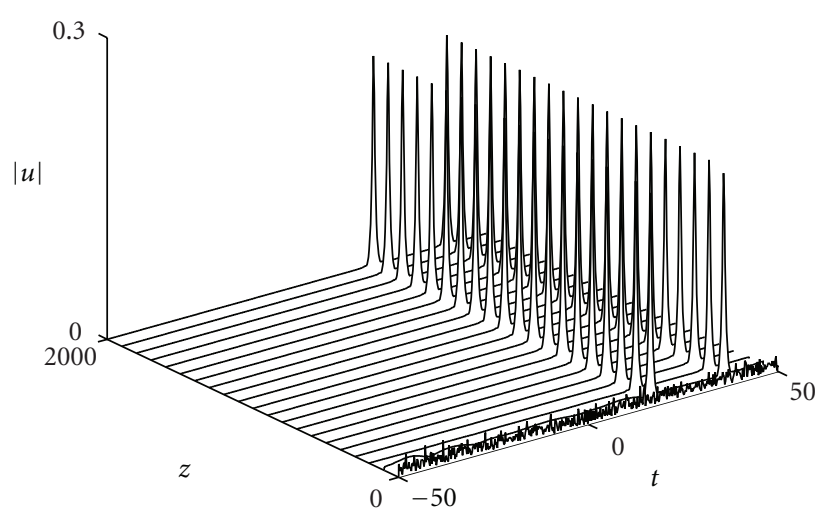

(c)

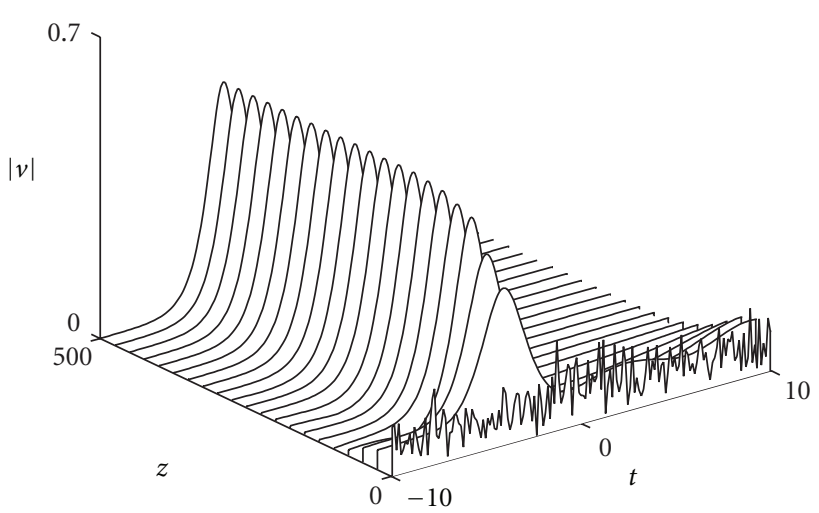

(b)

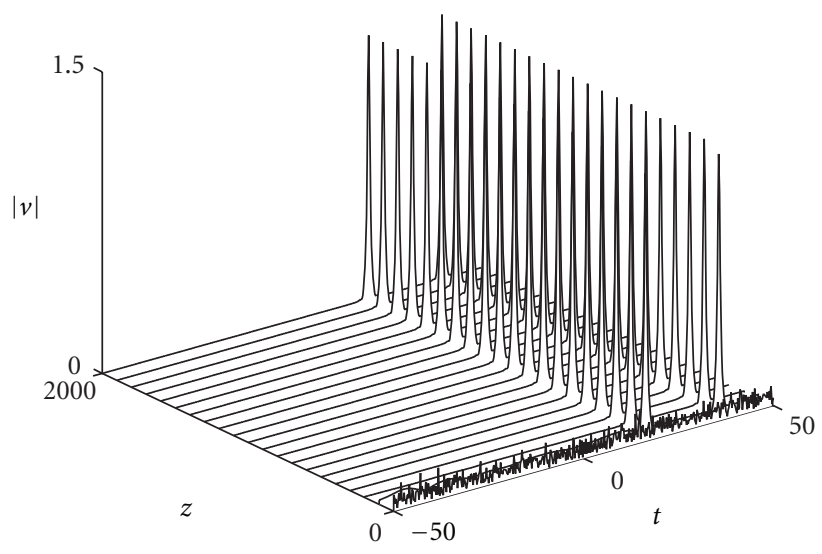

(d)

FIgURE 2: Self-starting mode-locking from white noise. (a) and (b) Stable single-pulse mode-locking when $g_{0}=1$. (c) and (d) Stable doublepulse mode-locking when $g_{0}=2.7$. The rest of the parameters are $D=0.4, K=0.1, e_{0}=1, \tau=0.1, \Gamma=0.1, \alpha_{1}=0, \alpha_{2}=0.82 \pi, \alpha_{3}=0.1 \pi$, and $\alpha_{p}=0.45 \pi$.

Here, $\psi$ represents the overall envelope of the electric field and the saturating gain is

$$
g(z)=\frac{2 g_{0}}{1+\left(1 / e_{0}\right) \int_{-\infty}^{\infty}|\psi|^{2} d t} .
$$

The derivation of the CQGLE that relates the coefficients to the experimental setup in Figure 1 was first given by Komarov et al. $[5,25]$. In the derivation they assumed that the orientations of the two quarter-waveplates add up to zero $\left(\alpha_{1}+\right.$ $\alpha_{2}=0$ ) and that the field is polarized along the fast component, that is, $\alpha_{p}=0$. Here, we will use a more general approach that can be found in $[6,29]$.

The key idea in deriving the CQGLE is to separate the linear and nonlinear effects in the CNLS (1), assuming that these effects occur on a length scale much longer than the fiber length $[5,6,25,29]$. We follow the circulating electric field in the laser cavity coming out from the polarizer. Ignoring the birefringence $K$ (which will be treated separately), the linear terms in (1) alone yield the evolution equation

$$
i \psi_{z}+\frac{D}{2} \psi_{t t}=i g(z)\left(1+\tau \partial_{t}^{2}\right) \psi-i \Gamma \psi
$$

The field envelope $\psi$ is related to the two orthogonally polarized components in the CNLS through the transformation $u=\psi \cos \alpha_{p}$ and $v=\psi \sin \alpha_{p}$, where $\alpha_{p}$ is the orientation of the polarizer. On the other hand, the nonlinear evolution

$$
\begin{aligned}
& i \frac{\partial u}{\partial z}+\left(|u|^{2}+A|v|^{2}\right) u+B v^{2} u^{*}=0 \\
& i \frac{\partial v}{\partial z}+\left(A|u|^{2}+|v|^{2}\right) v+B u^{2} v^{*}=0
\end{aligned}
$$

can be solved analytically to get

$$
\left(\begin{array}{l}
u^{-} \\
v^{-}
\end{array}\right)=e^{i I_{n}} J_{\mathrm{NL}}\left(\begin{array}{l}
u_{n} \\
v_{n}
\end{array}\right)=e^{i I_{n}}\left(\begin{array}{cc}
\cos w & \sin w \\
-\sin w & \cos w
\end{array}\right)\left(\begin{array}{l}
u_{n} \\
v_{n}
\end{array}\right),
$$

where $I_{n}=\left|u_{n}\right|^{2}+\left|v_{n}\right|^{2}$ is the total power of the field at the beginning of the fiber section during the $n$th round-trip and $w=B I_{n} \sin 2\left(\alpha_{1}-\alpha_{p}\right)$. The above map shows that the field undergoes an intensity-dependent polarization rotation (governed by $J_{\mathrm{NL}}$ ) as it propagates along the fiber. The effect of the fiber birefringence $K$ alone is

$$
i \frac{\partial u}{\partial z}-K u=0, \quad i \frac{\partial v}{\partial z}+K v=0,
$$


which leads to $u(z, t)=u(0, t) \exp (-i K z)$ and $v(z, t)=$ $v(0, t) \exp (i K z)$, respectively. In other words, the birefringence induces a $2 K$-phase shift between the two polarization components at the end of the fiber segment, that is, at $z=1$. Therefore, we approximate the birefringence using the matrix

$$
J_{K}=\left(\begin{array}{cc}
e^{-i K} & 0 \\
0 & e^{i K}
\end{array}\right)
$$

Applying this birefringent matrix and the Jones matrices given in (5) to (12) results in the scalar map

$$
\begin{aligned}
\psi_{n+1} & =e^{i\left|\psi_{n}\right|^{2}} Q\left(I_{n}\right) \psi_{n}, \\
& =e^{i\left|\psi_{n}\right|^{2}+\log Q} \psi_{n},
\end{aligned}
$$

where the complex function $Q$ is given by

$$
\begin{aligned}
Q=\frac{1}{2}\{ & e^{-i K}\left[\cos \left(2 \alpha_{2}-2 \alpha_{3}-\alpha_{p}\right)+i \cos \left(2 \alpha_{3}-\alpha_{p}\right)\right] \\
& \times\left[i \cos \left(2 \alpha_{1}-\alpha_{p}-w\right)-\cos \left(\alpha_{p}-w\right)\right] \\
& +e^{i K}\left[\sin \left(2 \alpha_{2}-2 \alpha_{3}-\alpha_{p}\right)-i \sin \left(2 \alpha_{3}-\alpha_{p}\right)\right] \\
& \left.\times\left[\sin \left(\alpha_{p}-w\right)-i \sin \left(2 \alpha_{1}-\alpha_{p}-w\right)\right]\right\}
\end{aligned}
$$

This complex quantity is explicitly related to the birefringence as well as the settings of the waveplates and polarizer. Equations (10) and (15) are the leading order approximation to the pulse propagation in the laser cavity shown in Figure 1. The initial data is first evolved forward over one round trip according to the linear equation (10). The discrete, nonlinear map (15) is then applied to the resulting field and the process is repeated.

The effect of the nonlinear transfer function $Q$ can be averaged into the evolution by taking a continuous limit of (15), yielding the differential equation [6]

$$
\psi_{z}=\left(i|\psi|^{2}+\log Q\left(|\psi|^{2}\right)\right) \psi
$$

The leading order approximation to the overall evolution is obtained by combining (10) and (17), that is,

$$
i \psi_{z}+\frac{D}{2} \psi_{t t}+|\psi|^{2} \psi=i g(z)\left(1+\tau \partial_{t}^{2}\right) \psi-i \Gamma \psi+i \log (Q) \psi
$$

We refer to this equation as the sinusoidal Ginzburg-Landau equation (SGLE) [29]. The coefficients in the CQGLE (8) can be related to the experimental settings by expanding the logarithmic term in the SGLE as power series in $|\psi|^{2}$ (assuming it is small) and truncating at the term $|\psi|^{4}$. Specifically, the CQGLE coefficients are calculated as

$$
\begin{aligned}
& \delta=\Gamma-\log |Q(0)|, \\
& \gamma=1+\operatorname{Im}\left(Q^{\prime}(0) / Q(0)\right), \\
& \beta=\operatorname{Re}\left(Q^{\prime}(0) / Q(0)\right), \\
& \nu=\operatorname{Im}\left[\left(Q(0) Q^{\prime \prime}(0)-Q^{\prime 2}(0)\right) / Q^{2}(0)\right] / 2, \\
& \mu=\operatorname{Re}\left[\left(Q(0) Q^{\prime \prime}(0)-Q^{\prime 2}(0)\right) / Q^{2}(0)\right] / 2,
\end{aligned}
$$

where the derivatives are taken with respect to $|\psi|^{2}$. In the case of the master mode-locking model (CGLE), the quintic coefficients $\nu$ and $\mu$ are set to zero. When the parameters are appropriately chosen, the CQGLE model supports solitary wave solutions that are known as dissipative solitons in the optics community [17]. It should be noted that in what follows the term soliton is used in a much broader sense than the strict mathematical definition of the localized solution of a completely integrable nonlinear evolution equation [41]. The form of the dissipative soliton is solely determined by the system parameters rather than by the initial condition as in the classical soliton theory.

In the CGLE, one has to adjust the orientations of the waveplates and polarizer in order that the cubic dissipation $\beta$ is always positive so that self-amplitude modulation (intensity discrimination) is possible. There is, however, only a small range of $\beta$ values that allows stable modelocking to occur $[42,43]$. Outside this range, the cubic gain is either too low for pulse formation or too large so that the pulse amplitude blows up to infinity after several cavity round trips. In reality, the pulse governed by the full equations (1) and (5) can never blow up since it always experiences a net loss upon passing through the modelocking elements (waveplates and polarizer). The CQGLE prevents the pulse from blowing up by saturating the cubic gain with a quintic loss $(\mu<0)$. This is a better description of the saturable absorption process, which makes the CQGLE a physically more relevant model than the master modelocking equation. In the regime where both the cubic and quintic dissipations are positive, there is no higher-order saturation in the model to prevent the blow-up of pulses, and thus the CQGLE becomes suspect as it admits a large number of unphysical behaviors. Therefore, the condition

$$
\beta>0>\mu
$$

is considered for the CQGLE to be a physically relevant model that does not exhibit blow-up. With an appropriate choice of parameters, the real part of the transfer function $Q$ provides an effective intensity discriminating mechanism (saturable absorption) to shape the circulating field. In this process the saturable absorber sifts out those intensities whose polarization state is commensurate with the orientation of the waveplates and polarizer, thus forming a stable mode-locked pulse. Figure 3 shows the experimentally (Figure 3(a)) and numerically predicted (Figure 3(b)) operating regimes of the laser depicted in Figure 1. In 


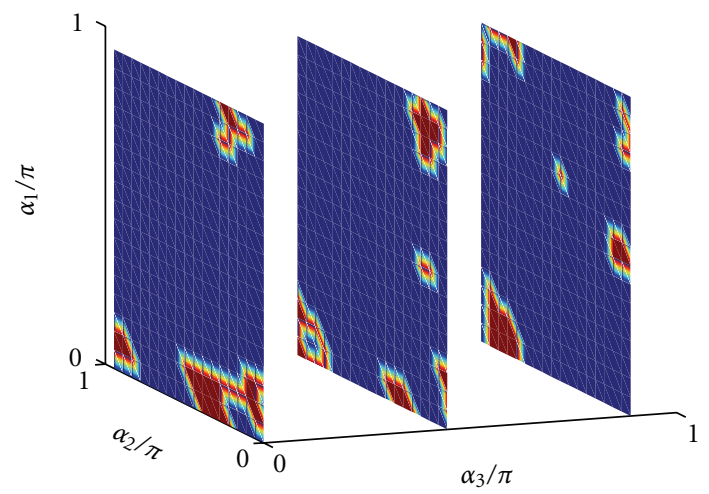

(a)

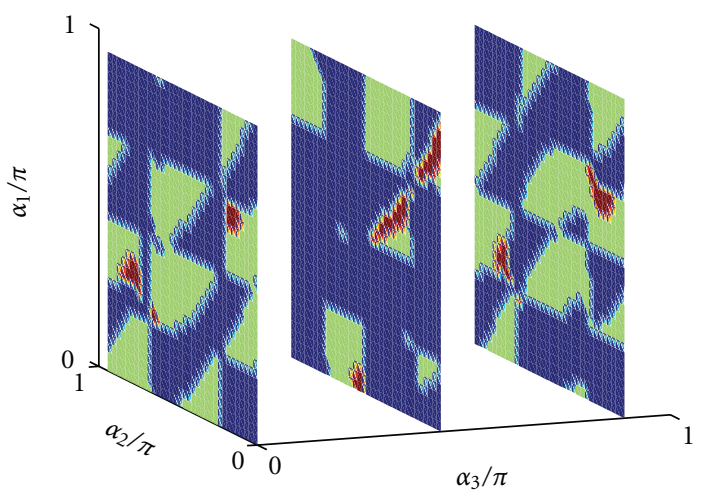

(b)

FIGURE 3: (a) Experimental operating regimes (red) of the laser shown in Figure 1. (b) Predicted operating regimes (red) of the CQGLE (8). The green region satisfies condition (20) but no stable pulses are found. Here $B=1 / 3, K=0.1, g_{0}=\delta, D=-0.3, \tau=0.2$, and $\alpha_{p}=0.45 \pi$ (from [6]).

the numerical result, mode-locking is only achievable in the red region. The green region satisfies condition (20), but stable mode-locked pulses cannot be found. This suggests that condition (20) alone is not sufficient to guarantee modelocking. A quantitative comparison between experiment and theory is difficult as the birefringence $K$ and the orientation of the fast axis of the fiber are hard to measure in practice. However, the theory reproduces the islands of stable regions, which are a key feature that is also observed in experiments.

\section{Dissipative Soliton Resonance}

When more energy is injected into the laser cavity, the modelocked pulse splits into multiple pulses (see Figure 2) instead of becoming a more intense single pulse. When the full set of cavity parameters that includes chromatic dispersion is taken into account, it is possible to find a certain region in the laser cavity vast parameter space for which the modelocked pulse becomes wider instead of splitting into multiple pulses as the cavity energy is increased. This specific method of using the cavity dispersion to circumvent MPI is referred to as dissipative soliton resonance (DSR) [44-46].

The CQGLE (8) is the standard theoretical model describing the averaged pulse dynamics in a ring cavity laser [ 1 , $3,5,6,37]$. Previous studies on DSR were phenomenological while the parameters in the CQGLE were chosen randomly. Comparing to the previous works on DSR $[45,46]$, the results here present two important additional features that pave the way for experimental investigations [44]. First, the parameters in the governing model are explicitly related to the waveplate/polarizer angles through expressions (19), allowing for a connection between theory and experiment. Second, a saturable, instead of a constant, gain is studied. This provides a more physically realistic picture that takes into account the finite pumping power budget $[1,3]$.

Without loss of generality, the self-phase modulation $\gamma$ in the CQGLE (8) can be normalized to one. Figure 4 shows the normalized cubic-quintic nonlinearities as functions of the quarter-waveplate angle $\alpha_{1}$ while the other parameters are fixed. Mode-locking dynamics, and in particular DSR, will be explored in regions where $\beta>0>\mu$ (see condition (20)). Other regions are considered as physically irrelevant since either the pulse will experience a blow-up in amplitude, or there is a lack of intensity discrimination [6]. The saturating gain is considered here, namely,

$$
g=\frac{2 g_{0}}{1+\|\psi\|^{2} / e_{0}} .
$$

This is a more physically realizable model than the constant gain model since the pump cannot maintain a fixed gain at arbitrary high cavity energy. In fact, it has been shown that the pulse solution of the master mode-locking equation (CQGLE with $\nu=\mu=0$ ) with constant gain is always unstable [27, 28, 42, 43].

In order to investigate the energy limiting effects of the saturable gain dynamics on the DSR, we use both $D$ and the saturating energy $e_{0}$ as control parameters with the results summarized in Figure 5. Usually for a fixed dispersion, the mode-locked pulse will become unstable when $e_{0}$ is too large. Such an instability is usually characterized by a Hopf bifurcation and marks the onset of MPI [24]. The cavity dispersion $D$ is the crucial factor in determining the dominant effect in the competition between DSR and MPI. Specifically, there is a critical limit $D=D_{c}$ such that the system favors DSR when $D<D_{c}$, and MPI when $D>D_{c}$. For the parameters considered in Figure 5, we found (numerically) that $D_{c} \approx-1.2$. Consider, for instance, the case where $D=$ $-1.6<D_{c}$. The Gaussian-looking pulse (blue solid curve) at low $e_{0}$ values first grows in amplitude until a saturating amplitude is reached (red dashed curve) and then in width to form a high-energy, flat-top structure (green dash-dot curve) at high $e_{0}$ values. The observed transformations in pulse shape and the chirp profile are signatures of the DSR although now infinite pulse energy cannot be achieved due to the finite saturation energy $e_{0}$. However, the physically unrealistic infinite pulse energy solutions of the constant gain DSR $[45,46]$ were key for motivating the present work with saturating, finite energy behavior. Remarkably, the onset of MPI is not observed even when the saturating energy is 


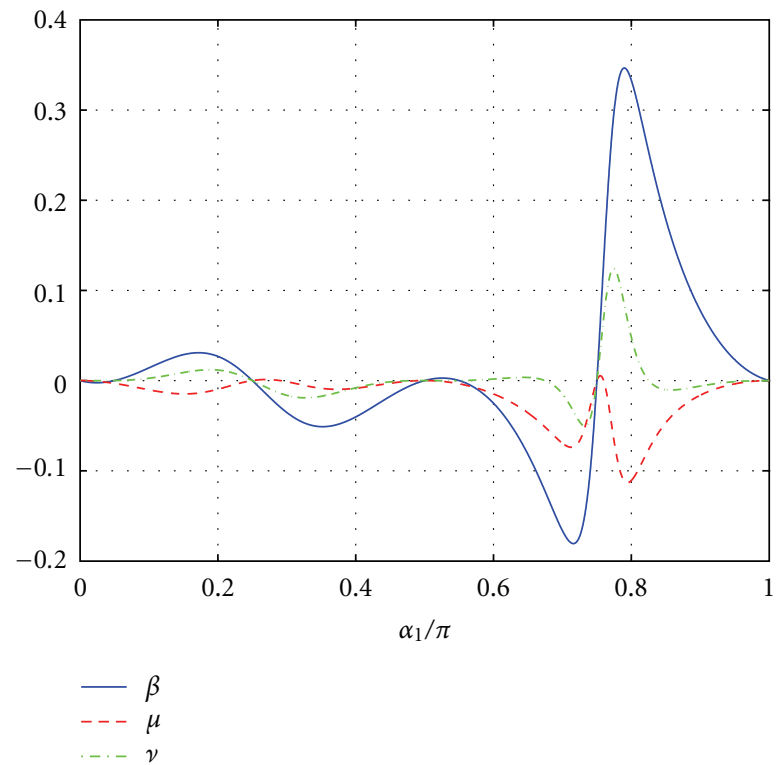

Figure 4: Normalized coefficients of the CQGLE as a function of $\alpha_{1}$ at $\alpha_{2}=0.16 \pi, \alpha_{3}=0.63 \pi, \alpha_{p}=0$, and $K=0.1$ (from [44]).

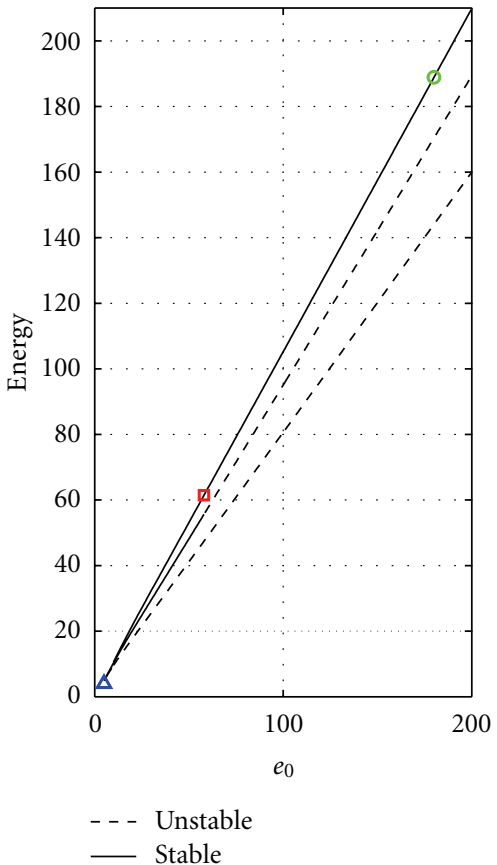

(a)
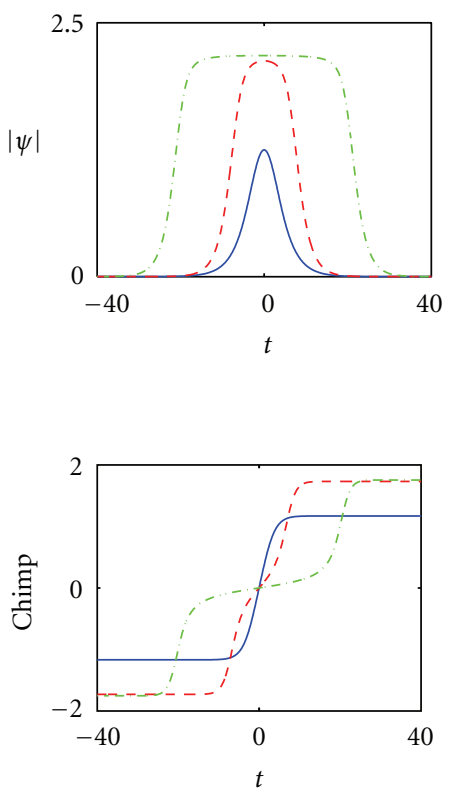

(b)

FIGURE 5: DSR in the case of saturating gain. (a) Pulse energy $\|\psi\|^{2}$ as a function of $e_{0}$ at $D=-1.6$ (top), $D=-1.2$ (middle), and $D=-0.8$ (bottom), respectively. The rest of the parameters are $g_{0}=2.3991, \tau=0.1667, \alpha_{1}=0.7863 \pi, \alpha_{2}=0.3 \pi, \alpha_{3}=\alpha_{p}=0, K=0.1$, and $\Gamma=0.1$. (b) The corresponding pulse shape (top) and frequency chirp profile (bottom) at $e_{0}=5$ (blue solid curves), $e_{0}=58$ (red dashed curves), and $e_{0}=180$ (green dash-dot curves) along the $D=-1.6$ line, respectively (from [44]).

as large as $e_{0}=200$. On the other hand, the Gaussianlooking mode-locked pulse becomes unstable long before the formation of the high-energy, flat-top pulse when $D=$ $-0.8>D_{c}$. The simulations here show that DSR can be used as an effective mechanism to circumvent MPI, provided the dispersion $D$ is appropriately chosen.

\section{Periodic Transmission: The Sinusoidal Ginzburg-Landau Equation}

It was shown in Section 2.2 that the CQGLE (8) can be obtained from the sinusoidal Ginzburg-Landau equation (18) by Taylor expanding the logarithmic term with $Q\left(|\psi|^{2}\right)$ 


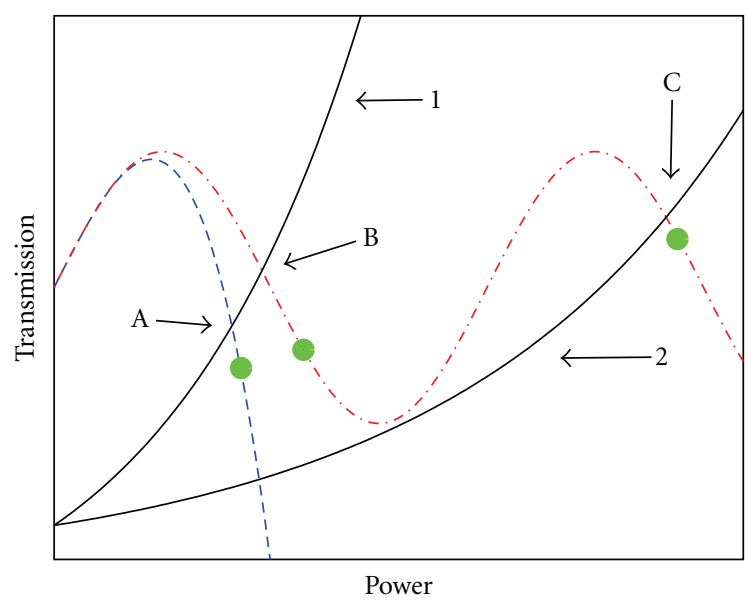

FIGURE 6: The red dash-dot line and the blue dashed line represent the transmission of the SGLE and the CQGLE, respectively. The black solid lines denote the saturating gain curves. The intersection of the gain and transmission curves represents a stable mode-locked pulse. The green circles are the maximum power allowed in the laser cavity. When energy is increased, gain curve 1 shifts towards gain curve 2, causing the mode-locked pulses A and B to exceed the maximum power allowed. In the CQGLE, the solution jumps to the next most energetically favorable configuration of a double-pulse solution (not shown). A high-energy single-pulse solution (point C) can exist in the SGLE model (from [29], 2011, IEEE).

being the periodic transmission produced by the saturable absorber (waveplates and polarizer). One has to choose the waveplate/polarizer settings such that the cubic-quintic coefficients satisfy condition (20) in order for the model to be physically relevant. Specifically, $\beta>0$ provides an effective pulse shaping mechanism in the laser cavity and $\mu<0$ saturates the cubic growth when the pulse amplitude becomes too large [3,6]. Condition (20) is an artificial constraint that arises solely from mathematical considerations and is the byproduct of using a truncated Taylor series. However, a series representation of the transmission function can lead to the elimination of important high-energy solution branches since the fundamental periodic transmission function is destroyed by the finite truncation. This fact has motivated the incorporation of the full logarithmic term rather than using its Taylor series in the averaged model, that is, using the SGLE (18) as the governing equation in the laser cavity. We will show that this model is able to support high-energy pulses that are not captured by the CQGLE [29].

4.1. Principle of Operation. To illustrate the key idea of the SGLE model, consider the generic transmission curves shown in Figure 6, which summarizes the main findings in the recent work of Li et al. [24]. The red dash-dot line and the blue dashed line represent the typical transmission function of the SGLE and the CQGLE, respectively. The black solid lines, on the other hand, denote the saturating gain curves in the cavity $[1,24,29,47]$. The intersection of the gain curve and the transmission curve describes a mode-locked solution where the cavity energy is in equilibrium. The points $\mathrm{A}$ and $\mathrm{B}$ along the gain curves represent the single modelocked solution of the CQGLE and SGLE, respectively. When the saturation energy is increased, the power of the modelocked pulse acquires a larger value which in turn shifts gain curve 1 towards gain curve 2 [24]. Eventually the power of the mode-locked solution exceeds the maximum value allowed (the green circles) in the laser system as computed from numerical simulations. In the case of CQGLE, the solution jumps to the next most energetically favorable configuration of a multipulse solution (not shown). For the SGLE, however, the solution is expressed as a stable single high-energy pulse at the point $\mathrm{C}$, which is not captured by the CQGLE transmission.

The advantage of incorporating the full sinusoidal transmission in the mode-locking model is twofold. First, since the transmission is not approximated by series expressions, the SGLE is able to give a more accurate description of the underlying mode-locking dynamics. Second, as shown above, high-energy pulses are possible only when a full analysis of the sinusoidal transmission curve is used [24, 29]. Thus, the SGLE model can serve as a design tool to maximize the energy output by adjusting the orientations of the waveplates and polarizer. Although analytical results are nontrivial due to the complexity of the equation, the SGLE is relatively easy to analyze with efficient numerical algorithms and reduction techniques [48-50].

\subsection{Comparison of the SGLE with Different Mode-Locking} Models. In this section, we will first establish that the SGLE is a valid mode-locking model that exhibits the essential dynamics in the nonlinear polarization rotation laser. Then, we will show that it is able to reproduce the high-energy pulses of the full governing system, solutions which are precluded from the master mode-locking theory and the CQGLE model.

4.2.1. Stable Mode-Locking and Multipulsing. We first compare the mode-locking performance governed by the full lumped mode-locking system (1)-(6) (CNLS in conjunction with the Jones matrices) and the SGLE (18). Figure 7 


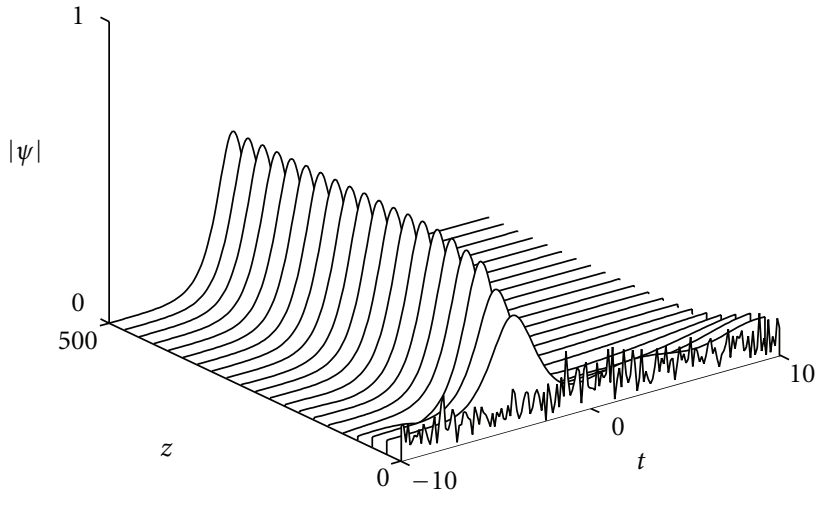

(a)

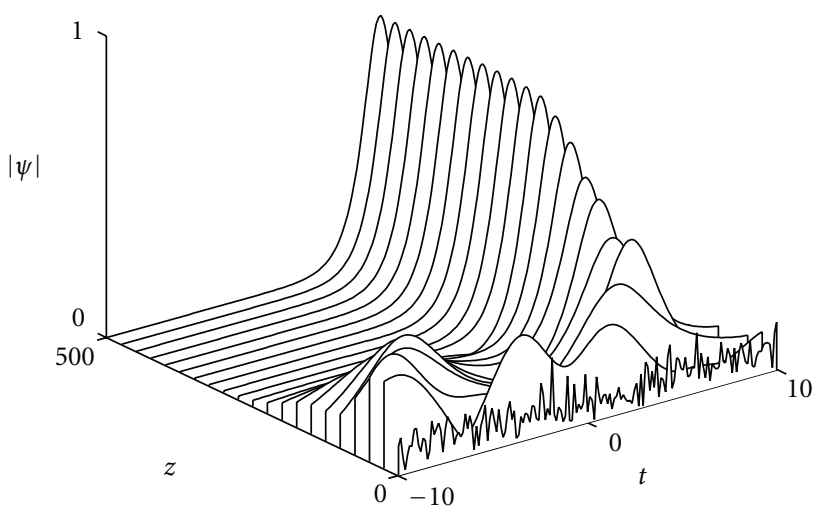

(c)

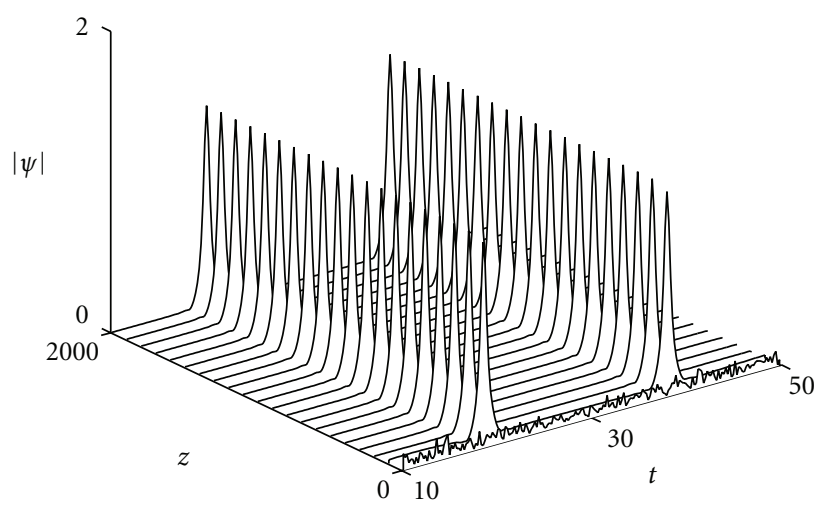

(b)

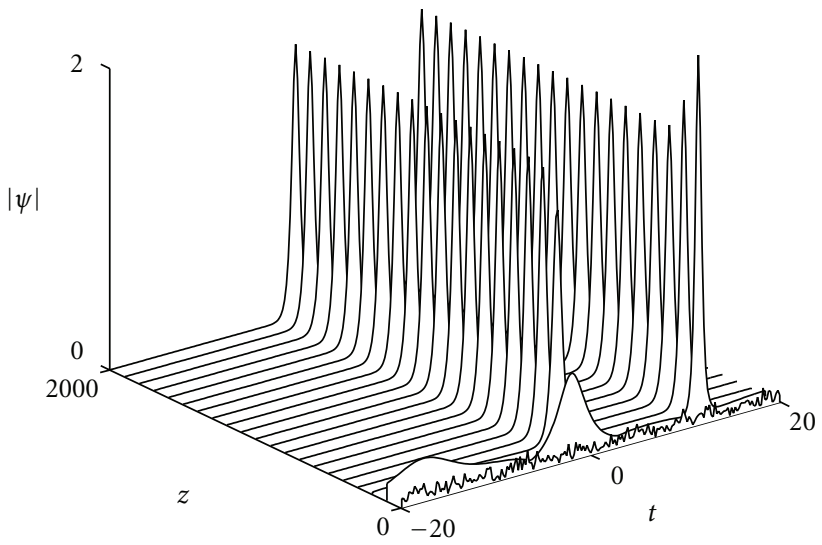

(d)

FIGURE 7: Numerical simulations of the full discrete governing system ((a) and (b), recall that $\left.|\psi|^{2}=|u|^{2}+|v|^{2}\right)$ and the SGLE ((c) and (d)) at different gain values $g_{0}$ in the anomalous dispersion regime. (a) and (c) Stable single-pulse evolution starting from white noise at $g_{0}=1$. (b) and (d) The initial white noise quickly evolves into two identical pulses per cavity round trip at $g_{0}=2.7$. The rest of the parameters in the simulations are $D=0.4, \alpha_{1}=0, \alpha_{2}=0.82 \pi, \alpha_{3}=0.1 \pi, \alpha_{p}=0.45 \pi, K=0.1, \Gamma=0.1, e_{0}=1$, and $\tau=0.1$ (from [29], 2011, IEEE).

demonstrates the self-starting behavior of the laser from a white noise initial condition in the anomalous dispersion regime $(D>0)$ with a particular waveplate/polarizer setting. In Figures $7(\mathrm{a})$ and $7(\mathrm{c})$, the pumping strength is set at $g_{0}=1$, and the initial white noise is dynamically locked into a stable stationary pulse after several hundred cavity round trips in both the full system (Figures $7(\mathrm{a})$ and $7(\mathrm{~b})$ ) and the SGLE (Figures 7(c) and 7(d)). The temporal location at which the pulse forms is completely arbitrary since random initial data is used. When the energy injected into the system is increased by increasing the gain $g_{0}$, the laser undergoes the commonly observed MPI [24]. In this situation, the initial condition quickly evolves into two or more pulses with identical energies, depending on the strength of the gain. It can be seen that the SGLE is capable of capturing qualitatively the MPI that occurs in the full system (Figures 7(b) and 7(d)) at a high gain level (e.g., $g_{0}=2.7$ ).
Qualitative matching between the SGLE model and full evolution is also achieved in the normal dispersion regime $(D<0)$, as shown in Figure 8. For the parameters considered, a single mode-locked pulse can be formed in both the full governing equations and the SGLE at $g_{0}=1$ (Figures 8(a) and $8(\mathrm{c})$ ). When the gain is increased to $g_{0}=3$, the laser cavity no longer supports a stable single-pulse solution and a double-pulse configuration is observed (Figures 8(b) and $8(d))$. From Figures 7 and 8, one can see that the two main differences between the full dynamics and the SGLE dynamics are (i) the duration of the transient evolution and (ii) the mode-locked amplitude. In the case of anomalous dispersion, for instance, it takes approximately 300 and 350 cavity round trips for the full system and the SGLE to modelock into a stable pulse, respectively. The mode-locked peak amplitude is 0.52 for the full model and 0.81 for the SGLE. The discrepancy is intrinsic for the averaged evolution 


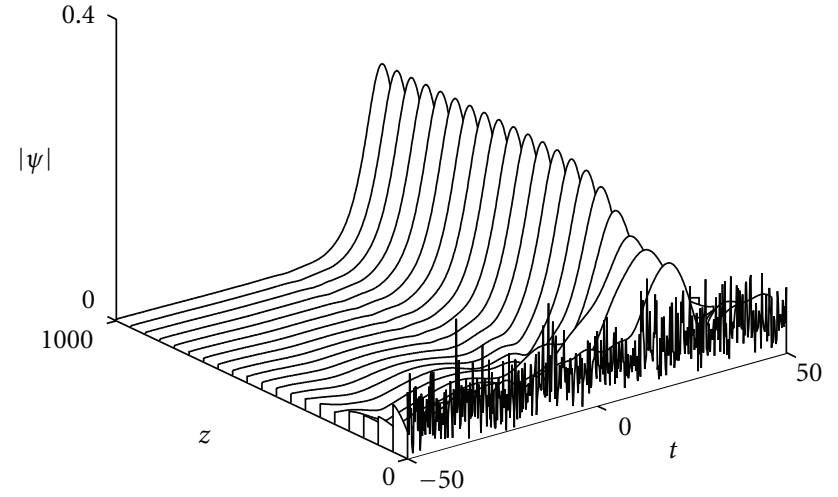

(a)

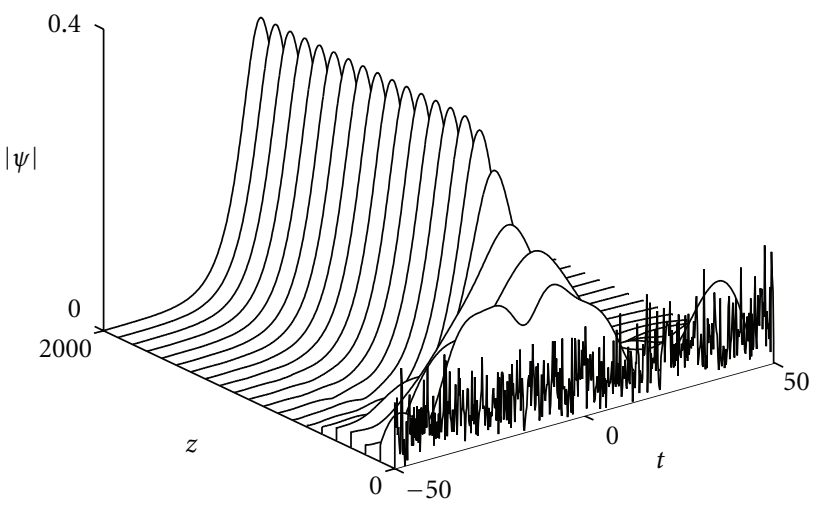

(c)

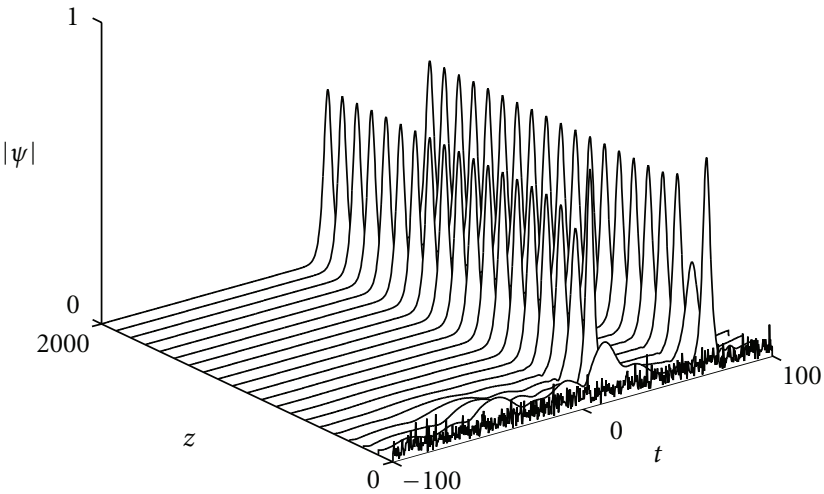

(b)

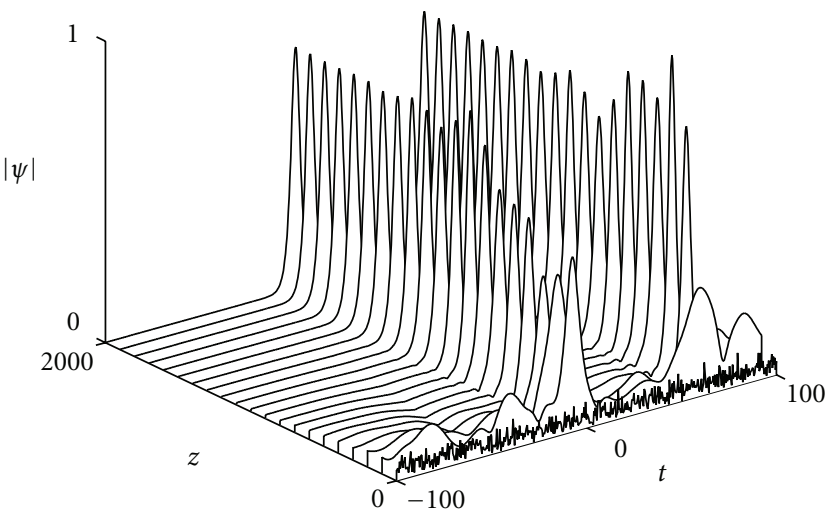

(d)

FIGURE 8: Numerical simulations of the full governing system ((a) and (b)) and the SGLE ((c) and (d)) at different gain values $g_{0}$ in the normal dispersion regime. (a) and (c) Stable single-pulse evolution starting from white noise at $g_{0}=1$. (b) and (d) The initial white noise evolves into two identical pulses per cavity round trip at $g_{0}=3$. The rest of the parameters in the simulations are $D=-0.4, \alpha_{1}=0.1 \pi$, $\alpha_{2}=0.554 \pi, \alpha_{3}=0.23 \pi, \alpha_{p}=0.43 \pi, K=0.1, \Gamma=0.1, e_{0}=1$, and $\tau=0.2$ (from [29], 2011, IEEE).

equations (including the CGLE and CQGLE). Nevertheless, this does not impact the usefulness of the SGLE model as it captures the self-starting nature of the laser and is easier to analyze than the full governing equations, that is, it is one scalar equation versus two coupled equations with discrete application of four Jones matrices.

4.2.2. Transition Dynamics. When the waveplate/polarizer angles $\alpha_{1}, \alpha_{2}, \alpha_{3}$, and $\alpha_{p}$ are chosen appropriately such that mode-locking is achievable, the initial data can evolve into an arbitrary number of stable pulses depending on the pumping strength $g_{0}$. In general, the transition from an $n$ pulse solution to an $(n+1)$-pulse solution is not a discrete process. Various types of transition dynamics can be obtained by modifying the waveplate/polarizer settings as well as other system parameters, as confirmed by theory and experiments $[24,51,52]$. Usually these transitional states are difficult to capture as they often happen in a small parameter regime, and fine tuning of the parameters is required to visualize them. Figure 9 shows a typical transition state of the laser cavity in the anomalous dispersion regime $(D>0)$. The pumping strength $g_{0}$ is chosen to be between the stable single- and double-pulse operations shown in Figure 7. Note that the $g_{0}$ values used in the full and SGLE models are different as one cannot expect an averaged or approximated model to match the original model at the exact same parameter values. The transitional state contains a tall pulse at the origin and one short pulse on each side of it. These side pulses are developed from the background. The entire structure undergoes small amplitude oscillations (at the order of $\left.10^{-4}\right)$. A remarkable agreement between the full governing equations (Figures $9(\mathrm{a})$ and $9(\mathrm{~b})$ ) and the SGLE mode (Figures $9(\mathrm{c})$ and $9(\mathrm{~d})$ ) is observed. This periodic state is stable and persists over long propagating distances. When 


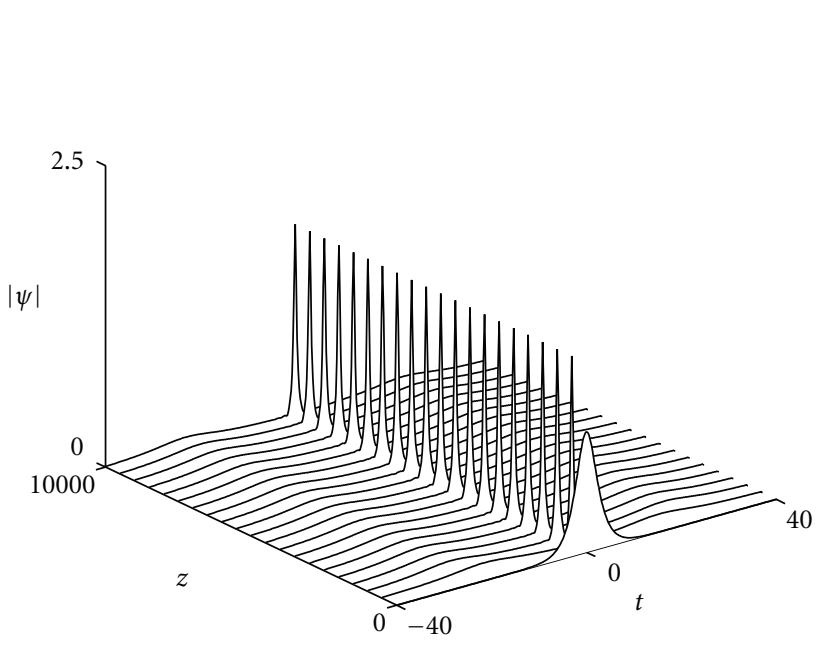

(a)

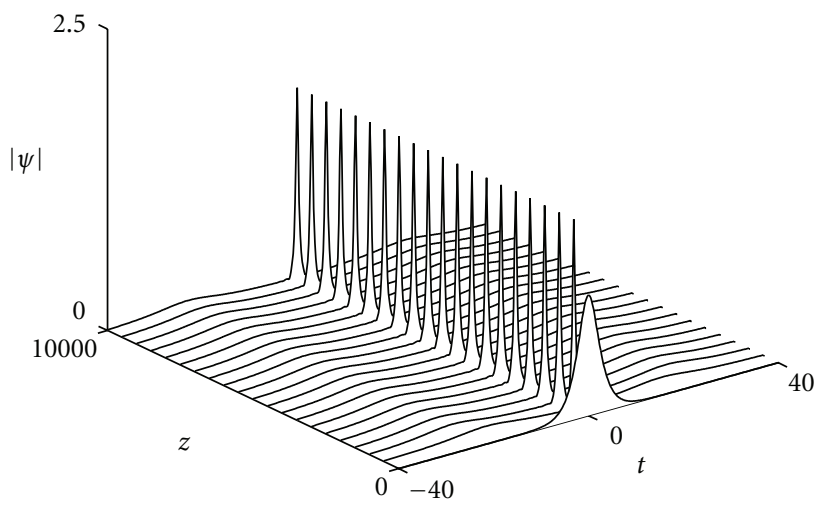

(c)

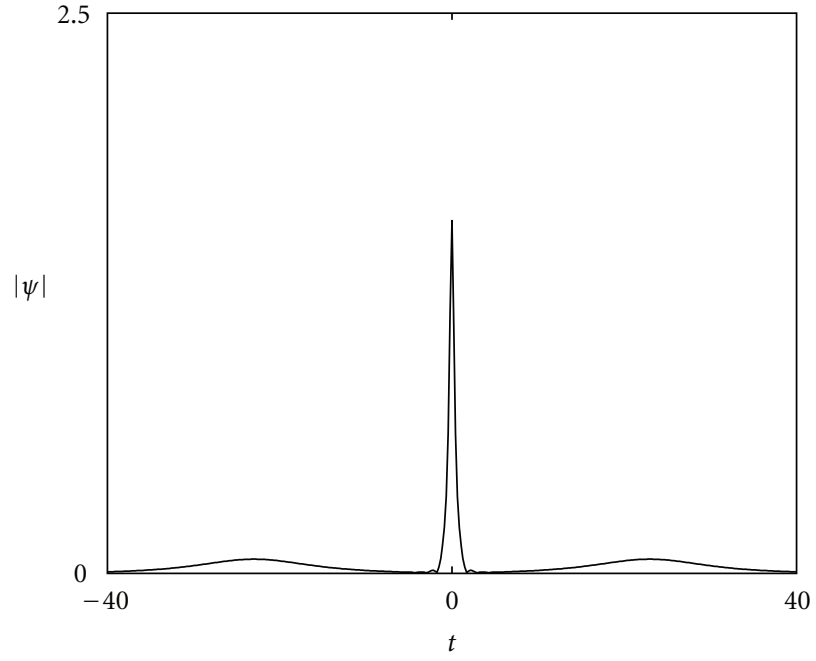

(b)

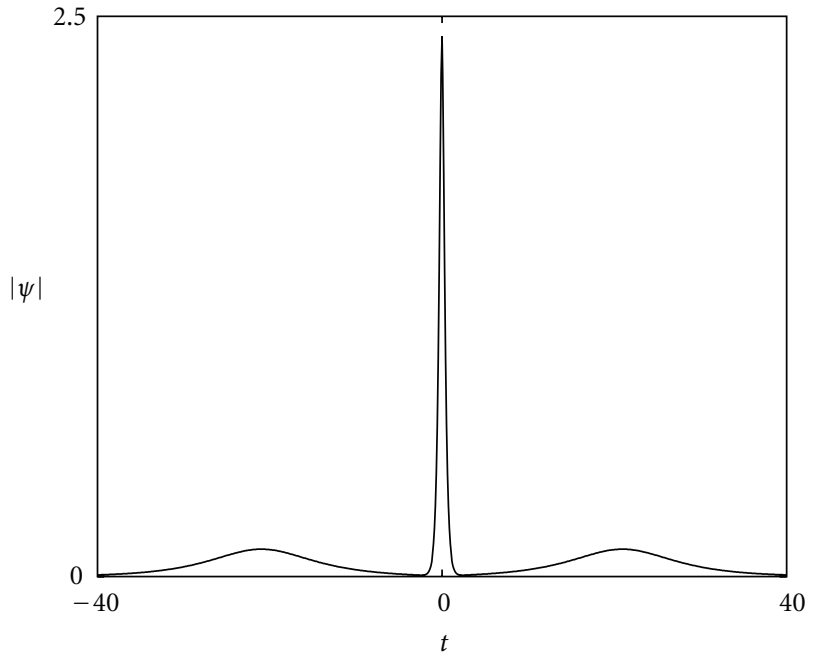

(d)

FIgURE 9: Transition dynamics observed in the anomalous dispersion regime. (a) and (b) Simulations of the full governing equations at $g_{0}=1.9((\mathrm{a})$ and $(\mathrm{c}))$ and the corresponding pulse profile ((b) and (d)). (c) and (d) Simulations of the SGLE at $g_{0}=2.25((\mathrm{a})$ and (c)) and the corresponding pulse profile ((b) and (d)). The rest of the parameters in the simulations are $D=0.4, \alpha_{1}=0, \alpha_{2}=0.82 \pi, \alpha_{3}=0.1 \pi$, $\alpha_{p}=0.45 \pi, K=0.1, \Gamma=0.1, e_{0}=1$, and $\tau=0.1$. The initial conditions in all the simulations are $\psi(0, t)=\operatorname{sech} 0.5 t$ (from [29], 2011, IEEE).

$g_{0}$ exceeds a critical value, the tall central pulse experiences a slight decrease in amplitude. At the same time, one of the side pulses is attenuated while the other one is amplified to the height of the central pulse, thus forming a double-pulse solution. The results found here match with those in a recent study on the CQGLE [49].

The transition dynamics are more subtle in the normal dispersion regime as depicted in Figure 10. Similar to the case of anomalous dispersion, a periodic structure with a tall central pulse and two flat side pulses is quickly developed (see Figures 10(a) and 10(b)). However, this periodic structure is unstable and eventually loses its stability after several thousand cavity round trips. A transient chaotic evolution is then observed followed by a stable copropagation of two well-separated pulses with different amplitudes. While the CQGLE (Figures 10(c) and 10(d)) is able to give a general qualitative approximation to the transitional state observed in the full simulation (Figures 10(a) and 10(b)), the SGLE model (Figures 10(e) and 10(f)) additionally gives a precise description of the transient chaotic behavior. This feature of the SGLE dynamics clearly demonstrates the improved description of the true discrete cavity dynamics.

4.2.3. Unphysical Blow-Up of Pulses. As mentioned in the beginning of the section, the field $\psi$ governed by the CQGLE may experience a blow-up with certain waveplate/polarizer settings when condition (20) is not satisfied. This blow-up is unphysical since energy is always lost when the electric field 


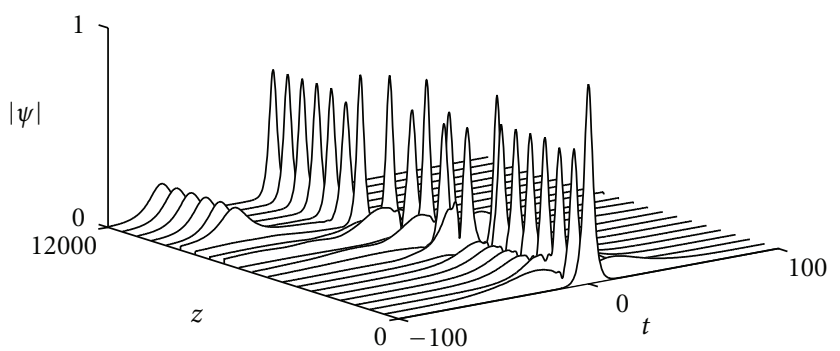

(a)

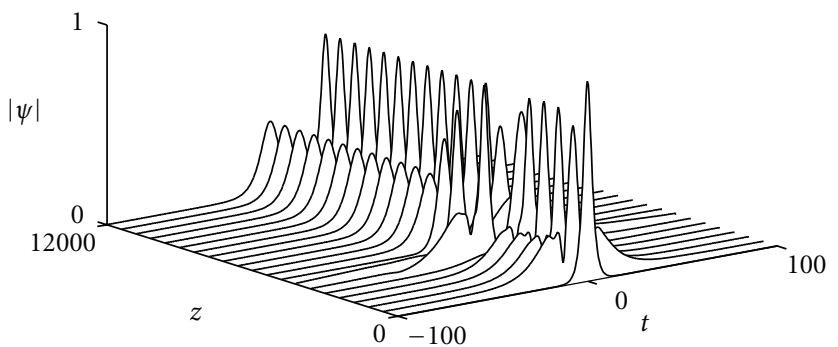

(c)

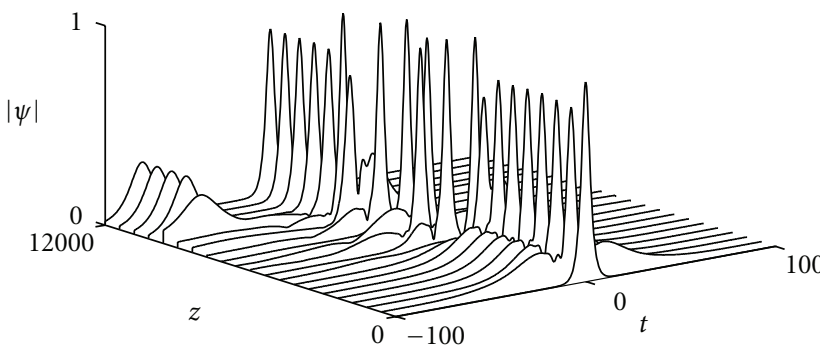

(e)

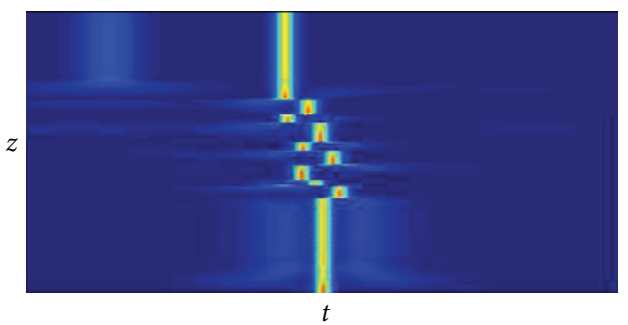

(b)

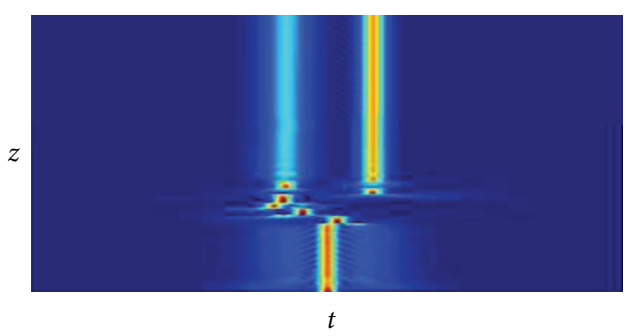

(d)

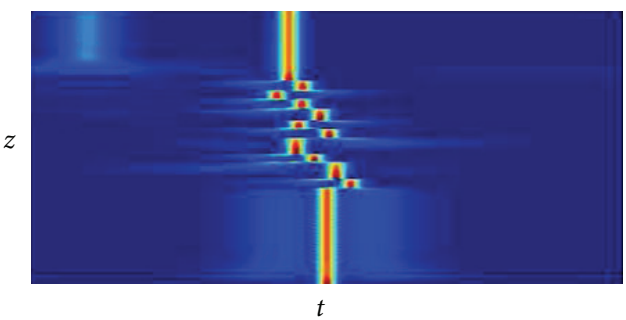

(f)

FIgURe 10: Periodic evolution observed in the full governing equations ((a) and (b), $\left.g_{0}=2.27\right)$, CQGLE ((c) and (d), $\left.g_{0}=2.2\right)$, and SGLE ((e) and (f), $\left.g_{0}=2.2\right)$. The simulated evolutions are shown in (a), (c), and (e) and the corresponding pseudocolor plots are shown in (b), (d), and (f). The rest of the parameters in the simulations are $D=-0.4, \alpha_{1}=0.1 \pi, \alpha_{2}=0.554 \pi, \alpha_{3}=0.23 \pi, \alpha_{p}=0.43 \pi, K=0.1, \Gamma=0.1$, $e_{0}=1$, and $\tau=0.2$. The initial conditions in all the simulations are $\psi(0, t)=\operatorname{sech} 0.5 t$ (from [29], 2011, IEEE).

passes through the mode-locking elements and is a result of the finite truncation of the periodic transmission function $\log Q$. It is our intent to show that the artificial restriction (20) is unnecessary for modeling the laser cavity.

To show that the SGLE does not have the above limitations, we study the transmission function $T\left(|\psi|^{2}\right)$ which can be defined as the sum of the linear loss and the nonlinear dissipation. The transmission function of the CQGLE $\left(T_{\mathrm{cq}}\right)$ and the SGLE $\left(T_{s}\right)$ are given by

$$
\begin{aligned}
& T_{\mathrm{cq}}\left(|\psi|^{2}\right)=-\delta+\beta|\psi|^{2}+\mu|\psi|^{4}, \\
& T_{s}\left(|\psi|^{2}\right)=-\Gamma+\operatorname{Re}\left(\log Q\left(|\psi|^{2}\right)\right) .
\end{aligned}
$$

In the last equation, the imaginary part of the logarithmic term is neglected since it is not responsible for intensity discrimination and energy transfer. The imaginary terms only act to shift the intensity-dependent index of refraction response due to the field intensity. Shown in Figure 11 are the transmission curves for the CQGLE (blue dotted line) and SGLE (red solid line) at two different quarter-waveplate angles (Figure 11(a): $\alpha_{2}=0.554 \pi$, Figure 11(b): $\alpha_{2}=0.4 \pi$ ). The transmission function $T_{\mathrm{cq}}$ of the CQGLE is quadratic in the field power $|\psi|^{2}$. It curves downward at $\alpha_{2}=0.554 \pi$ and upward at $\alpha_{2}=0.4 \pi$. Figure 12 illustrates the pulse evolutions for the full governing equations (Figures 12(a) and 12(b)), CQGLE (Figures 12(c) and 12(d)), and the SGLE (Figures 12(e) and 12(f)) corresponding to the two $\alpha_{2}$ values considered in Figure 11. At $\alpha_{2}=0.554 \pi$ (Figures 12(a), 


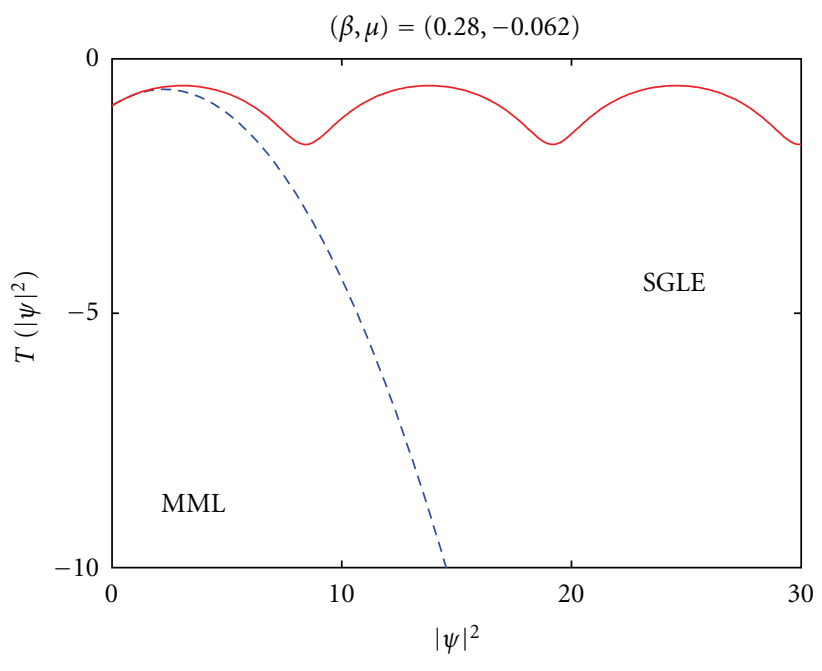

(a)

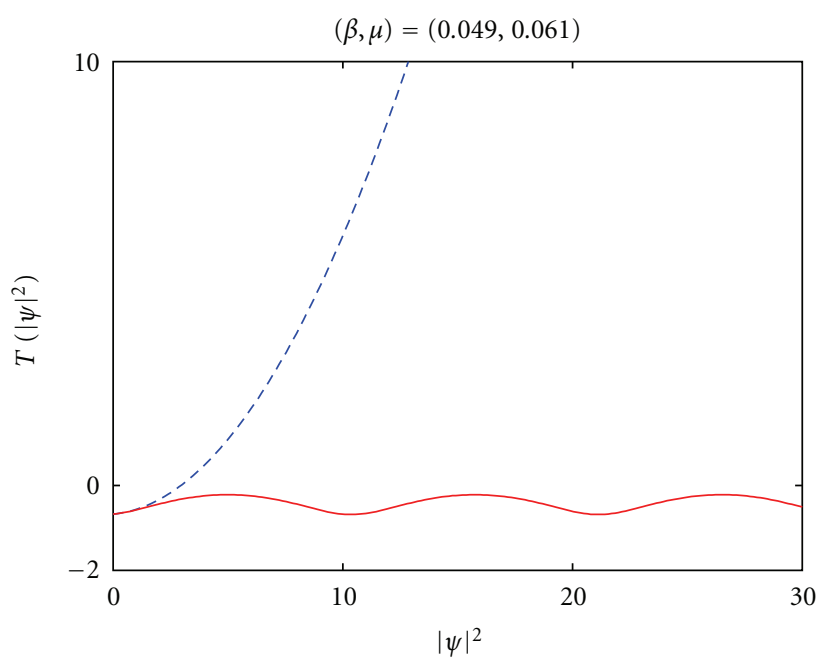

(b)

FIGURE 11: The transmission curve for the CQGLE (blue dashed line) and SGLE (red solid line) for $\alpha_{2}=0.554 \pi$ (a) and $\alpha_{2}=0.4 \pi$ (b). The rest of the parameters are $\alpha_{1}=0.1 \pi, \alpha_{3}=0.23 \pi, \alpha_{p}=0.43 \pi, \Gamma=0.1, e_{0}=1$, and $K=0.1$ (from [29], 2011, IEEE).

12(c), and 12(e)), the evolutions governed by all three models are always of finite amplitude. Blowing up of pulses is observed in the CQGLE at $\alpha_{2}=0.4 \pi$ since self-saturation is impossible when both the nonlinear coefficients $\beta$ and $\mu$ are positive (Figures 12(b), 12(d) and 12(f)). For both $\alpha_{2}$ values considered, the SGLE does not have the problem of unphysical blow-up since it incorporates the full transmission $\log Q$ induced by the waveplates and polarizer. This allows for a drastically broader and more realistic range of waveplate/polarizer settings for which stable solutions can be achieved.

4.2.4. High-Energy Mode-Locking. As illustrated in Figure 6, the CQGLE uses a quadratic polynomial in the field power $|\psi|^{2}$ (see (22)) to approximate the full sinusoidal transmission. This can severely restrict the maximum amplitude a single pulse can reach before it splits. Figure 13 shows the tallest possible stable pulse generated by the full governing equations (black dash-dot line), CQGLE (red dashed line), and SGLE (green solid line) at two different half-waveplate angles $\alpha_{3}$. These pulses are recorded right before the MPI occurs when the pumping strength $g_{0}$ exceeds the multipulsing threshold. At $\alpha_{3}=0.2 \pi$ (Figure 13(a)), the full and the SGLE models produce pulses with amplitudes of about 3.1. The total cavity energy, which is given by the $L^{2}$-norm

$$
E=\int_{-\infty}^{\infty}|\psi|^{2} d t
$$

is calculated for each model. For the SGLE, the limiting pumping strength $g_{0}=3.58$ gives a pulse energy of $E=4.52$. For the full model, the tallest pulse has an energy of $E=3$ with pumping strength of $g_{0}=3.38$. One can see that the limiting $g_{0}$ value for a stable single-pulse operation in the present case is higher than that for a double-pulse operation $\left(g_{0}=2.7\right)$ shown in Figure 7 . The energy confined in each individual pulse of the double-pulse solution shown in Figure 7 is $E=2.37$ for the SGLE and $E=1.11$ for the full governing equations, which is significantly less than the energy output in the present case. The limiting pumping strength for the CQGLE model is only $g_{0}=2.51$, and the resulting pulse does not match well with the full simulation and the SGLE since it is much shorter and wider. With the same set of parameters, the maximum energy in the singlepulse solution of the CQGLE is $E=2.87$, which is only approximately $60 \%$ of the SGLE model.

At $\alpha_{3}=0.25 \pi$ (Figure 13(b)), the maximum pulse amplitudes allowed in both the full and the SGLE models increase to about 4 . The corresponding pulse energies are $E=6.58\left(g_{0}=11.7\right)$ and $E=8.516\left(g_{0}=15.4\right)$, respectively, which are about twice the total energy of the double-pulse solutions shown in Figure 7. This is a remarkable achievement in terms of maximizing the energy output of the laser without going through multi pulsing. On the other hand, such a high-intensity pulse is not supported by the CQGLE with the parameters considered. In particular, expressions (19) reveal that the transmission $T_{\mathrm{cq}}$ of the CQGLE (c.f. (22)) is characterized by $\beta=-0.63$ and $\mu=5.74$. At high intensities, the quintic gain always dominates the cubic loss and consequently leads to an unphysical blow-up of the solution, as happens in this case.

The above simulations show that, indeed, the SGLE can support high-intensity pulses with enormous energies observed in the full governing system, including those that are unpredicted by the CQGLE model. Although these simulations are done with anomalous dispersion, similar trends are also observed in the normal dispersion regime. Given the vast parameter space of the SGLE, it is possible to obtain other types of interesting mode-locking dynamics such as soliton shaking and period doubling bifurcation [24, 53]. The ability to support, model, and characterize high-intensity and highenergy mode-locked solutions is the key reason for our development of the proposed SGLE theory. In the work 


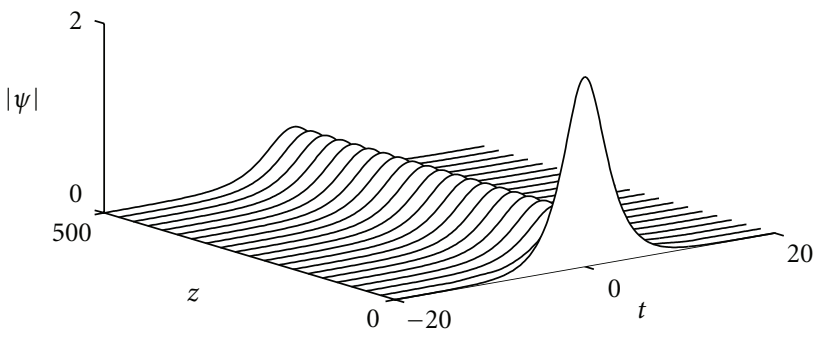

(a)

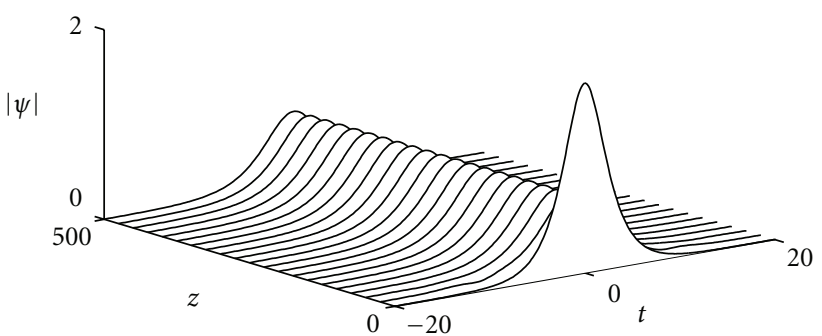

(c)

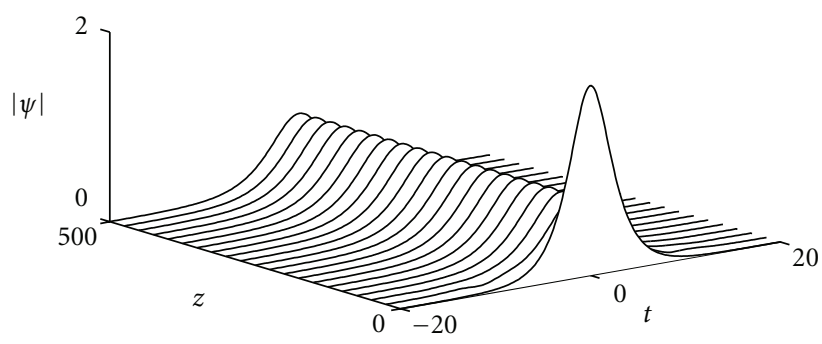

(e)

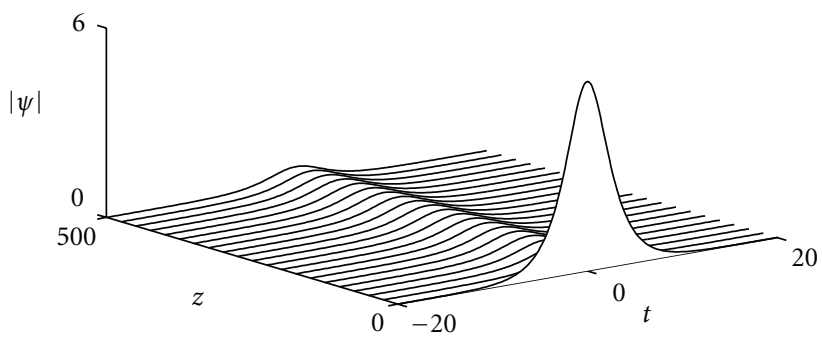

(b)

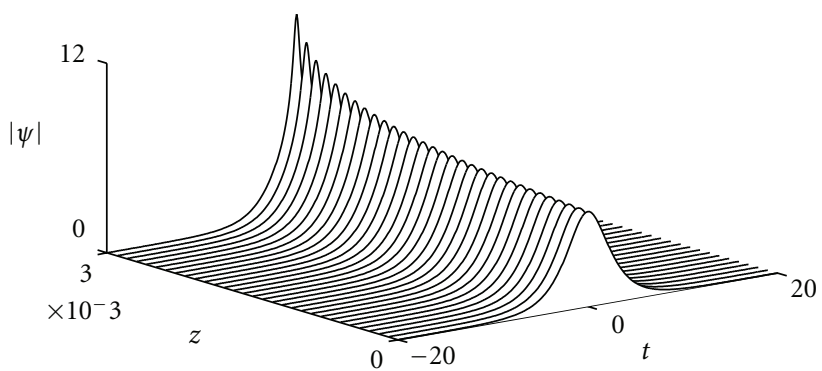

(d)

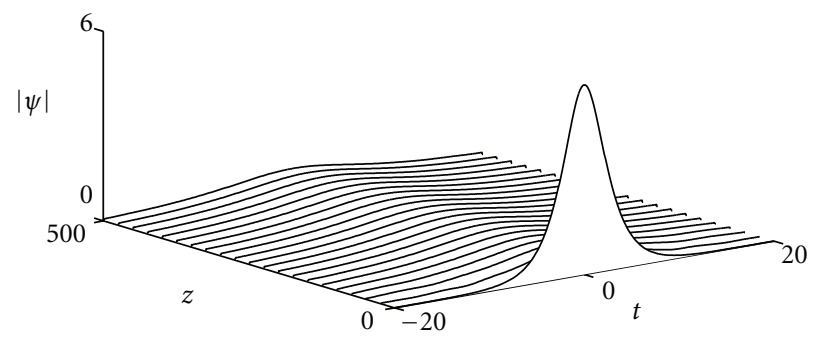

(f)

Figure 12: Pulse evolution predicted by the full governing equations ((a) and (b)), CQGLE ((c) and (d)), and SGLE ((e) and (f)). The plots on the left ((b), (d), and (f)) column correspond to the left (b) in Figure 11. The initial conditions for the plots on the left are $\psi(0, t)=$ 2 sech $0.5 t$, while those for the right plots are $\psi(0, t)=6$ sech $0.5 t$. The dispersion and pumping strength in all the simulations are $D=-0.4$ and $g_{0}=2$ (from [29], 2011, IEEE).

by Renninger et al. [37], it was found that a number of high-energy pulsed solutions existed in the CQGLE with both positive cubic $(\beta)$ and quintic $(\mu)$ nonlinearities. The SGLE discussed here may provide for an effective way to stabilize such high-energy solutions. Ultimately, it is critical in modern mode-locked lasers to understand how to achieve pulses with maximal energy. The SGLE theory provides a basis for exploring such pulses.

\section{Summary}

We have studied the phenomenon of dissipative soliton resonance (DSR) in the context of the CQGLE and have extended previous findings to coefficients that can be explicitly related to the settings of the ring cavity laser depicted in Figure 1. In addition to the constant gain model, which was studied previously $[45,46]$, DSR is also achievable in the physically relevant case of saturating gain, but it is subjected to the onset of MPI if the cavity is not carefully engineered. Specifically, we found that there is a critical normal cavity dispersion $D_{c}$ above which the DSR phenomenon is favored over MPI. Due to its linear chirp profile, the wide pulse created by DSR may be compressed effectively by linear dispersive delay lines, thus forming a short, intense pulse with enormous amount of energy.

In addition to varying the cavity dispersion $D$, high-energy mode-locked pulses can also be achieved by carefully adjusting the waveplate/polarizer settings, which in turn alters the characteristics of the sinusoidal transmission. The SGLE (sinusoidal Ginzburg-Landau equation) theory predicts that one can significantly increase the maximum pumping strength $g_{0}$ allowed before pulse splitting occurs. The resulting high-intensity single pulses can deliver significantly more energy than previous theoretical predictions by 


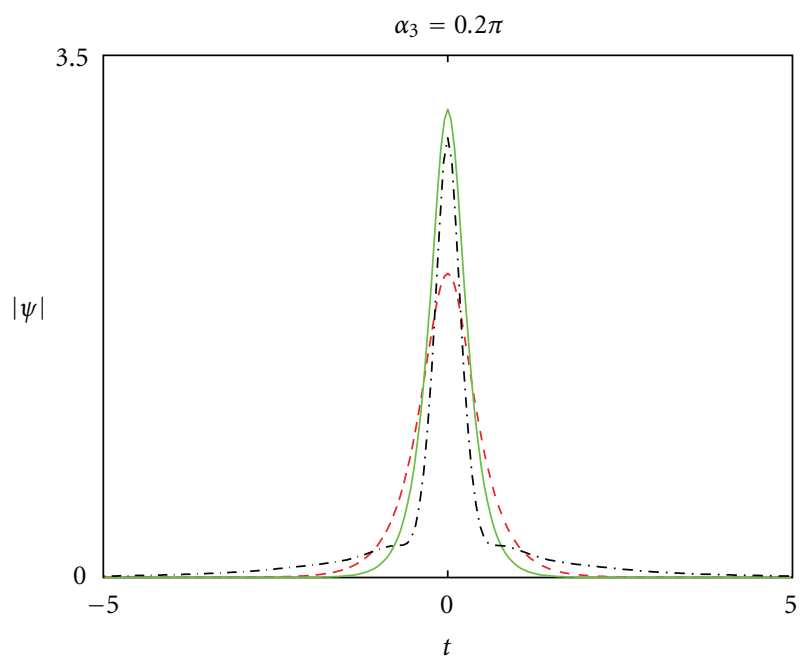

(a)

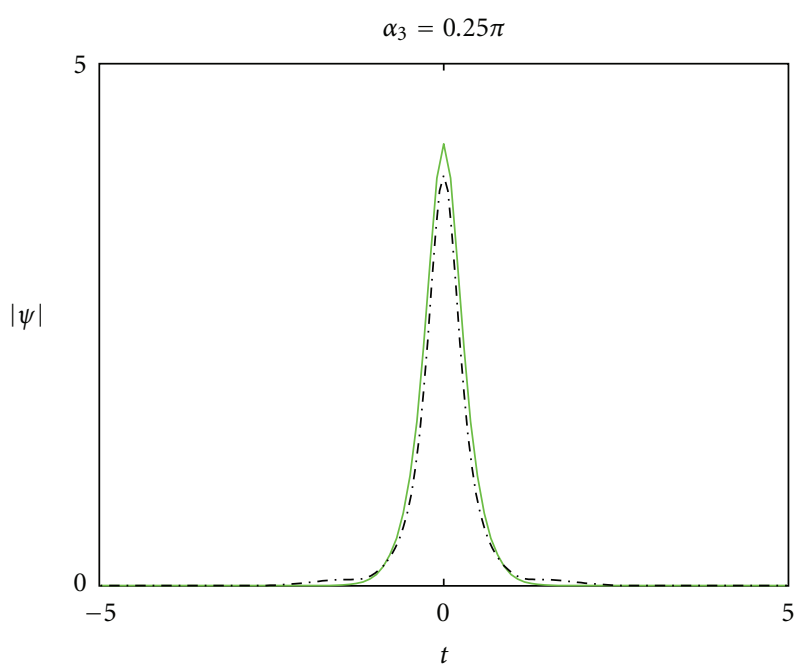

(b)

FIGURE 13: Stable single-pulse solution with the largest possible amplitude of the full governing system (black dash-dot curve), CQGLE (red dashed curve), and SGLE (green solid curve) at $\alpha_{3}=0.2 \pi$ (a) and $\alpha_{3}=0.25 \pi(\mathrm{b})$. The rest of the parameters are $D=0.4, \tau=0.1$, $e_{0}=1, \Gamma=0.1, \alpha_{1}=0, \alpha_{2}=0.49 \pi, \alpha_{p}=0.45 \pi$, and $K=0.1$. The initial conditions are $\psi(0, t)=0.5 \operatorname{sech} t$ (from [29], 2011, IEEE).

the conventional CQGLE and are thus practically important to high-power applications. These pulses can exist in the parameter regime that is not allowed by the existing theories [6]. Thus, the SGLE can be used as an excellent design tool for enhancing the energy output of the laser. The current theoretical treatment of DSR and periodic transmission illustrate how the orders of magnitude increase in pulse energy can be achieved in order to make fiber lasers directly competitive with leading solid state mode-locking configurations. The tremendous benefits of fiber lasers in terms of cost, readily available technology, and ease of use lend importance to the theoretical developments proposed in this dissertation. DSR in the context of the SGLE will be investigated in the future in order to bring the pulse energy to an even higher level.

\section{Acknowledgments}

J. N. Kutz acknowledges support from the National Science Foundation (NSF) (DMS-1007621) and the US Air Force Office of Scientific Research (AFOSR) (FA955009-0174). W. Renninger and F. Wise acknowledge the support by the National Science Foundation (ECCS0901323) and the National Institutes of Health (EB002019). Ph. Greulu acknowledges the support from the Agence Nationale de la Recherche (Grant ANR-2010-BLANC-041701-SOLICRISTAL).

\section{References}

[1] H. A. Haus, "Mode-locking of lasers," IEEE Journal on Selected Topics in Quantum Electronics, vol. 6, no. 6, pp. 1173-1185, 2000.

[2] I. N. Duling III and M. L. Dennis, Compact Sources of Ultrashort Pulses, Cambridge University Press, 1995.

[3] J. N. Kutz, "Mode-locked soliton lasers," SIAM Review, vol. 48, no. 4, pp. 629-678, 2006.

[4] C. Spielmann, P. F. Curley, T. Brabec, E. Winter, and F. Krausz, "Generation of sub-20 fs mode-locked pulses from Ti:sapphire laser," Electronics Letters, vol. 28, no. 16, pp. 1532-1534, 1992.

[5] A. Komarov, H. Leblond, and F. Sanchez, "Multistability and hysteresis phenomena in passively mode-locked fiber lasers," Physical Review A, vol. 71, no. 5, Article ID 053809, 9 pages, 2005.

[6] E. Ding and J. N. Kutz, "Operating regimes, split-step modeling, and the Haus master mode-locking model," Journal of the Optical Society of America B, vol. 26, no. 12, pp. 2290-2300, 2009.

[7] H. A. Haus, J. G. Fujimoto, and E. P. Ippen, "Analytic theory of additive pulse and Kerr lens mode locking," IEEE Journal of Quantum Electronics, vol. 28, no. 10, pp. 2086-2096, 1992.

[8] K. Tamura, H. A. Haus, and E. P. Ippen, "Self-starting additive pulse mode-locked erbium fibre ring laser," Electronics Letters, vol. 28, no. 24, pp. 2226-2228, 1992.

[9] H. A. Haus, E. P. Ippen, and K. Tamura, "Additive-pulse modelocking in fiber lasers," IEEE Journal of Quantum Electronics, vol. 30, no. 1, pp. 200-208, 1994.

[10] M. E. Fermann, M. J. Andrejco, Y. Silberberg, and M. L. Stock, "Passive mode-locking by using nonlinear polarization evolution in a polarizing-maintaining erbiumdoped fiber laser," Optics Letters, vol. 29, p. 447, 1993.

[11] I. N. Duling, "Subpicosecond all-fibre erbium laser," Electronics Letters, vol. 27, no. 6, pp. 544-545, 1991.

[12] D. J. Richardson, R. I. Laming, D. N. Payne, V. J. Matsas, and M. W. Phillips, "Self-starting, passively modelocked erbium fiber laser based on the amplifying Sagnac switch," Electronics Letters, vol. 27, p. 542, 1991.

[13] M. L. Dennis and I. N. Duling, "High repetition rate figure eight laser with extracavity feedback," Electronics Letters, vol. 28, no. 20, pp. 1894-1896, 1992.

[14] F. Ö. Ilday, F. W. Wise, and T. Sosnowski, "High-energy femtosecond stretched-pulse fiber laser with a nonlinear optical loop mirror," Optics Letters, vol. 27, no. 17, pp. 1531-1533, 2002. 
[15] Q. Bao, H. Zhang, Y. Wang et al., "Atomic-layer craphene as a saturable absorber for ultrafast pulsed lasers," Advanced Functional Materials, vol. 19, no. 19, pp. 3077-3083, 2009.

[16] H. Zhang, D. Tang, R. J. Knize, L. Zhao, Q. Bao, and K. P. Loh, "Graphene mode locked, wavelength-tunable, dissipative soliton fiber laser," Applied Physics Letters, vol. 96, no. 11, Article ID 111112, 3 pages, 2010.

[17] N. N. Akhmediev and A. Ankiewicz, Dissipative Solitons, Springer, 2005.

[18] J. L. Proctor and J. N. Kutz, "Passive mode-locking by use of waveguide arrays," Optics Letters, vol. 30, no. 15, pp. 20132015, 2005.

[19] J. Proctor and J. N. Kutz, "Nonlinear mode-coupling for passive mode-locking: application of waveguide arrays, dualcore fibers, and/or fiber arrays," Optics Express, vol. 13, no. 22, pp. 8933-8950, 2005.

[20] K. Intrachat and J. N. Kutz, "Theory and simulation of passive modelocking dynamics using a long-period fiber grating," IEEE Journal of Quantum Electronics, vol. 39, no. 12, pp. 15721578, 2003.

[21] A. Chong, J. Buckley, W. Renninger, and F. Wise, "All-normaldispersion femtosecond fiber laser," Optics Express, vol. 14, no. 21, pp. 10095-10100, 2006.

[22] A. Chong, W. H. Renninger, and F. W. Wise, "All-normal-dispersion femtosecond fiber laser with pulse energy above $20 \mathrm{~nJ}$," Optics Letters, vol. 32, no. 16, pp. 2408-2410, 2007.

[23] A. Chong, W. H. Renninger, and F. W. Wise, "Properties of normal-dispersion femtosecond fiber lasers," Journal of the Optical Society of America B, vol. 25, no. 2, pp. 140-148, 2008.

[24] F. Li, P. K. A. Wai, and J. N. Kutz, "Geometrical description of the onset of multipulsing in mode-locked laser cavities," Journal of the Optical Society of America B, vol. 27, no. 10, pp. 2068-2077, 2010.

[25] A. Komarov, H. Leblond, and F. Sanchez, "Quintic complex Ginzburg-Landau model for ring fiber lasers," Physical Review E, vol. 72, no. 2, Article ID 025604, 4 pages, 2005.

[26] B. C. Collings, S. T. Cundiff, N. N. Akhmediev, J. M. SotoCrespo, K. Bergman, and W. H. Knox, "Polarization-locked temporal vector solitons in a fiber laser: experiment," Journal of the Optical Society of America B, vol. 17, no. 3, pp. 354-365, 2000.

[27] J. M. Soto-Crespo, N. N. Akhmediev, and V. V. Afanasjev, "Stability of the pulselike solutions of the quintic complex Ginzburg-Landau equation," Journal of the Optical Society of America B, vol. 13, no. 7, pp. 1439-1449, 1996.

[28] J. M. Soto-Crespo, N. N. Akhmediev, V. V. Afanasjev, and S. Wabnitz, "Pulse solutions of the cubic-quintic complex Ginzburg-Landau equation in the case of normal dispersion," Physical Review E, vol. 55, no. 4, pp. 4783-4796, 1997.

[29] E. Ding, E. Shlizerman, and J. N. Kutz, "Generalized master equation for high-energy passive mode-locking: the sinusoidal Ginzburg-Landau equation," IEEE Journal of Quantum Electronics, vol. 47, no. 5, pp. 705-714, 2011.

[30] G. P. Agrawal, Nonlinear Fiber Optics, Academic Press, 2001.

[31] A. Hasegawa, Optical Solitons in Fibers, Springer, 2003.

[32] C. R. Menyuk, "Nonlinear pulse propagation in birefringent optical fibers," IEEE Journal of Quantum Electronics, vol. 23, no. 2, pp. 174-176, 1987.

[33] C. R. Menyuk, "Pulse propagation in an elliptically birefringent Kerr medium," IEEE Journal of Quantum Electronics, vol. 25, no. 12, pp. 2674-2682, 1989.

[34] D. Y. Tang, W. S. Man, and H. Y. Tam, "Stimulated soliton pulse formation and its mechanism in a passively mode-locked fibre soliton laser," Optics Communications, vol. 165, no. 4, pp. 189-194, 1999.

[35] D. Y. Tang, L. M. Zhao, B. Zhao, and A. Q. Liu, "Mechanism of multisoliton formation and soliton energy quantization in passively mode-locked fiber lasers," Physical Review A, vol. 72, no. 4, Article ID 043816, 9 pages, 2005.

[36] D. Y. Tang, W. S. Man, H. Y. Tam, and P. D. Drummond, "Observation of bound states of solitons in a passively mode-locked fiber laser," Physical Review A, vol. 64, no. 3, Article ID 033814, 3 pages, 2001.

[37] W. H. Renninger, A. Chong, and F. W. Wise, "Dissipative solitons in normal-dispersion fiber lasers," Physical Review A, vol. 77, no. 2, Article ID 023814, 4 pages, 2008.

[38] P. E. Langridge, G. S. McDonald, W. J. Firth, and S. Wabnitz, "Self-sustained mode locking using induced nonlinear birefringence in optical fibre," Optics Communications, vol. 97, no. 3-4, pp. 178-182, 1993.

[39] H. Leblond, M. Salhi, A. Hideur, T. Chartier, M. Brunel, and F. Sanchez, "Experimental and theoretical study of the passively mode-locked ytterbium-doped double-clad fiber laser," Physical Review A, vol. 65, no. 6, Article ID 063811, 9 pages, 2002.

[40] M. Salhi, H. Leblond, and F. Sanchez, "Theoretical study of the stretched-pulse erbium-doped fiber laser," Physical Review A, vol. 68, no. 3, Article ID 033815, 7 pages, 2003.

[41] M. J. Ablowitz and H. Segur, Solitons, Nonlinear Evolution Equations and Inverse Scattering, Cambridge University Press, New York, NY, USA, 1991.

[42] T. Kapitula, J. N. Kutz, and B. Sandstede, "Stability of pulses in the master mode-locking equation," Journal of the Optical Society of America B, vol. 19, no. 4, pp. 740-746, 2002.

[43] T. Kapitula, N. Kutz, and B. Sandstede, "The Evans function for nonlocal equations," Indiana University Mathematics Journal, vol. 53, no. 4, pp. 1095-1126, 2004.

[44] E. Ding, P. Grelu, and J. N. Kutz, "Dissipative soliton resonance in a passively mode-locked fiber laser," Optics Letters, vol. 36, no. 7, pp. 1146-1148, 2011.

[45] P. Grelu, W. Chang, A. Ankiewicz, J. M. Soto-Crespo, and N. Akhmediev, "Dissipative soliton resonance as a guideline for high-energy pulse laser oscillators," Journal of the Optical Society of America B, vol. 27, no. 11, pp. 2336-2341, 2010.

[46] N. Akhmediev, J. M. Soto-Crespo, and P. Grelu, "Roadmap to ultra-short record high-energy pulses out of laser oscillators," Physics Letters, Section A, vol. 372, no. 17, pp. 3124-3128, 2008.

[47] S. Namiki, E. P. Ippen, H. A. Haus, and C. X. Yu, "Energy rate equations for mode-locked lasers," Journal of the Optical Society of America B, vol. 14, no. 8, pp. 2099-2111, 1997.

[48] C. R. Jones and J. N. Kutz, "Stability of mode-locked pulse solutions subject to saturable gain: computing linear stability with the Floquet-Fourier-Hill method," Journal of the Optical Society of America B, vol. 27, no. 6, pp. 1184-1194, 2010.

[49] E. Ding, E. Shlizerman, and J. N. Kutz, "Modeling multipulsing transition in ring cavity lasers with proper orthogonal decomposition," Physical Review A, vol. 82, no. 2, Article ID 023823, 10 pages, 2010.

[50] B. G. Bale and J. N. Kutz, "Variational method for modelocked lasers," Journal of the Optical Society of America B, vol. 25, no. 7, pp. 1193-1202, 2008.

[51] B. G. Bale, K. Kieu, J. N. Kutz, and F. Wise, "Transition dynamics for multi-pulsing in mode-locked lasers," Optics Express, vol. 17, no. 25, pp. 23137-23146, 2009. 
[52] Q. Xing, L. Chai, W. Zhang, and C. Y. Wang, "Regular, perioddoubling, quasi-periodic, and chaotic behavior in a self-modelocked Ti:sapphire laser," Optics Communications, vol. 162, no. 1, pp. 71-74, 1999.

[53] J. M. Soto-Crespo, P. Grelu, N. Akhmediev, and N. Devine, "Soliton complexes in dissipative systems: vibrating, shaking, and mixed soliton pairs," Physical Review E, vol. 75, no. 1, Article ID 016613, 9 pages, 2007. 

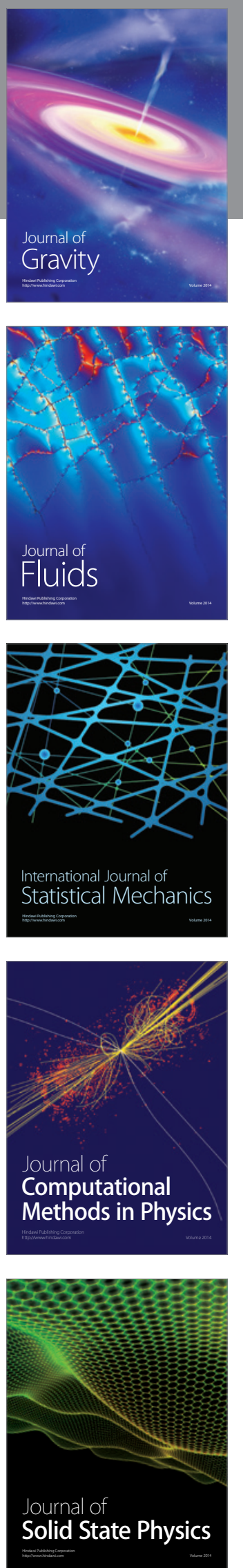

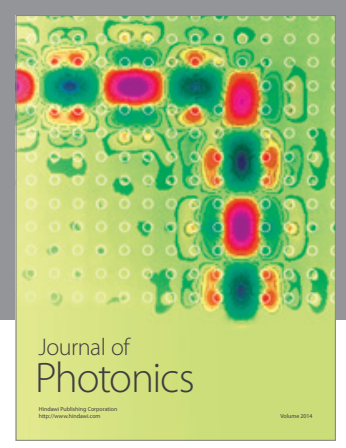

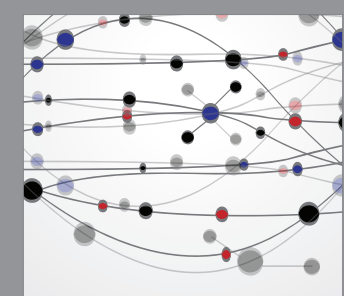

The Scientific World Journal
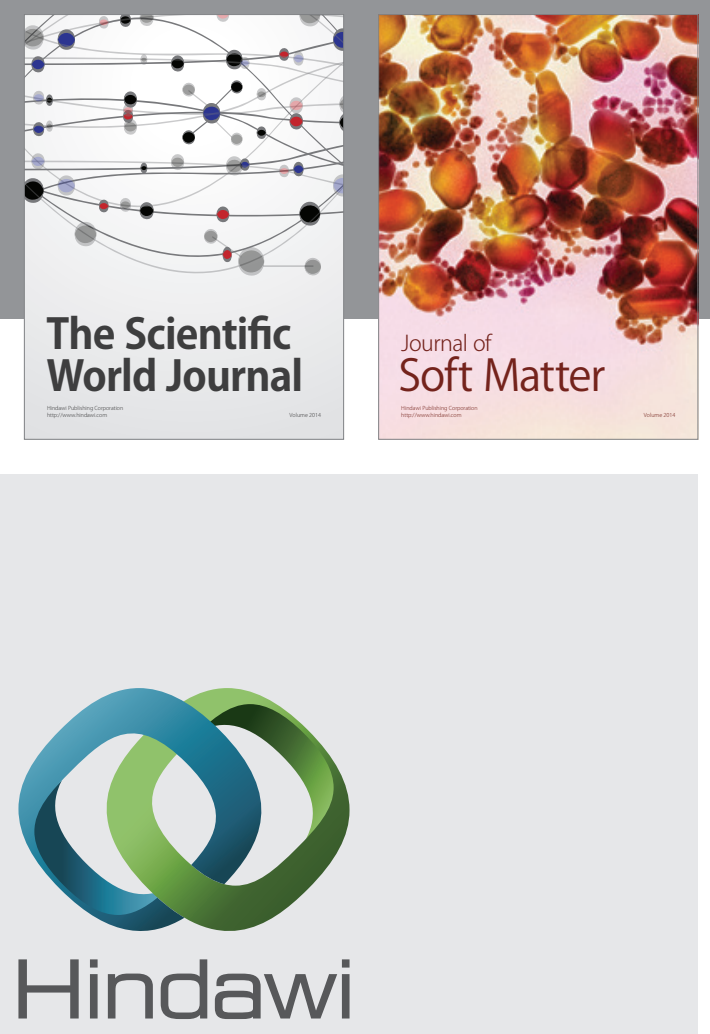

Submit your manuscripts at

http://www.hindawi.com
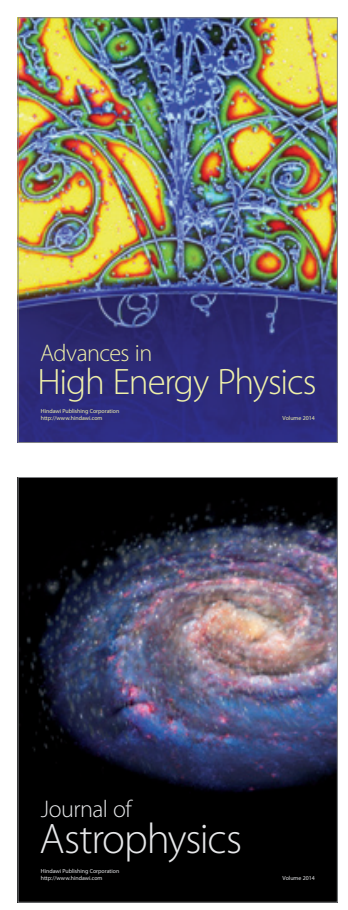
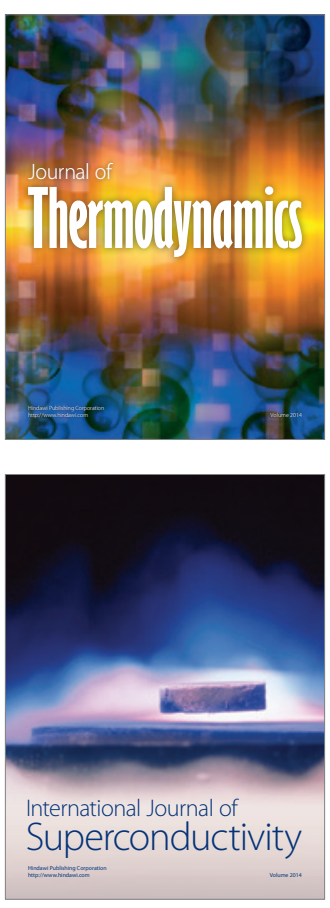
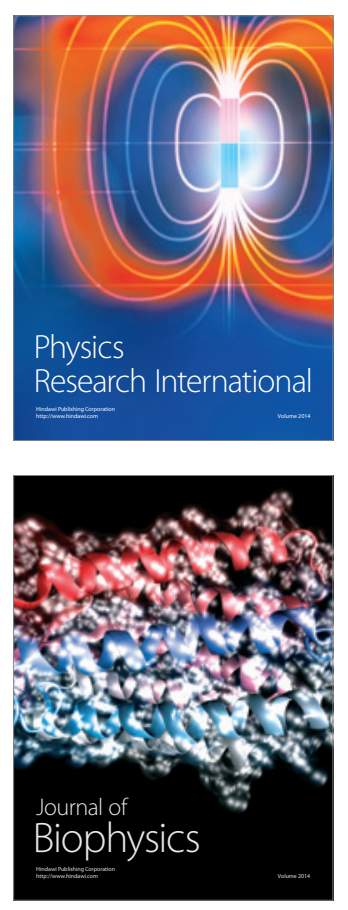
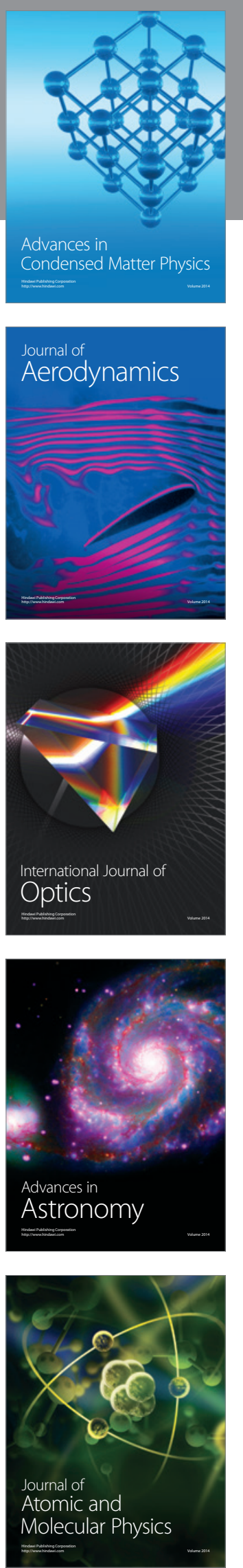